\title{
Non-Gaussian Correlations between Reflected and Transmitted Intensity Patterns Emerging from Opaque Disordered Media
}

\author{
I. Starshynov, ${ }^{1}$ A. M. Paniagua-Diaz, ${ }^{1}$ N. Fayard,${ }^{2}$ A. Goetschy, ${ }^{2}$ R. Pierrat, ${ }^{2}$ R. Carminati, ${ }^{2, *}$ and J. Bertolotti ${ }^{1, \dagger}$ \\ ${ }^{1}$ University of Exeter, Stocker Road, Exeter EX4 4QL, United Kingdom \\ ${ }^{2}$ ESPCI Paris, PSL Research University, CNRS, Institut Langevin, 1 Rue Jussieu, F-75005 Paris, France
}

(Received 1 December 2017; revised manuscript received 8 March 2018; published 11 May 2018)

\begin{abstract}
The propagation of monochromatic light through a scattering medium produces speckle patterns in reflection and transmission, and the apparent randomness of these patterns prevents direct imaging through thick turbid media. Yet, since elastic multiple scattering is fundamentally a linear and deterministic process, information is not lost but distributed among many degrees of freedom that can be resolved and manipulated. Here, we demonstrate experimentally that the reflected and transmitted speckle patterns are robustly correlated, and we unravel all the complex and unexpected features of this fundamentally nonGaussian and long-range correlation. In particular, we show that it is preserved even for opaque media with thickness much larger than the scattering mean free path, proving that information survives the multiple scattering process and can be recovered. The existence of correlations between the two sides of a scattering medium opens up new possibilities for the control of transmitted light without any feedback from the target side, but using only information gathered from the reflected speckle.
\end{abstract}

DOI: 10.1103/PhysRevX.8.021041

\section{INTRODUCTION}

In multiply scattering materials, the random inhomogeneities in the refractive index scramble the incident wavefront, mixing colors and spatial degrees of freedom, resulting in a white and opaque appearance [1]. Under illumination with coherent light and for elastic scattering, interference produces large intensity fluctuations that are not averaged out by a single realization of the disorder, resulting in a seemingly random speckle pattern [2]. In principle, the speckle pattern encodes all the information on the sample and the incident light [3], and complete knowledge of the scattering matrix allows one to reverse the multiple scattering process and to recover the initial wavefront, thus permitting imaging through turbid materials $[4,5]$.

Speckle patterns are not as random as they appear at first sight. Interference between the possible scattering paths in the medium is known to produce spatial correlations between the intensity measured at different positions

\footnotetext{
* Corresponding author. remi.carminati@espci.fr

Corresponding author. j.bertolotti@exeter.ac.uk

Published by the American Physical Society under the terms of the Creative Commons Attribution 4.0 International license. Further distribution of this work must maintain attribution to the author(s) and the published article's title, journal citation, and DOI.
}

Subject Areas: Complex Systems, Mesoscopics, Photonics

[6-8], and correlations of different ranges have been identified [9]. Short-range correlations determine the size of a speckle spot, while long-range correlations emerge as a consequence of constraints such as energy conservation or reciprocity [10-12]. The idea of using spatial correlations for imaging has recently emerged $[13,14]$, but it has been so far limited to the optical memory effect [15], a correlation of purely geometrical origin.

At first glance, as transmitted and reflected waves are expected to undergo very different multiple scattering sequences, correlations between transmitted and reflected wavefronts are expected to quickly average to zero. Very little attention has been given to the cross-correlation between the intensities measured at two points on opposite sides of the scattering medium (i.e., in the reflected and transmitted speckles patterns), and their existence has only been mentioned in passing [16,17]. However, a recent theoretical study suggested that a long-range correlation should survive even for thick (opaque) scattering media [18]. The existence of this reflection-transmission (R-T) correlation suggests that one could noninvasively extract information on the transmitted speckle from a measurement restricted to the reflection half-space. As the discovery of new speckle correlations, like the recently described shift memory effect [19], has been systematically translated into novel imaging techniques in the past [20], we suggest that the reflection-transmission correlation we describe here will be of fundamental importance for future developments 
of applications based on reflection measurements, such as in vivo biological imaging [21].

In this work, we identify the nontrivial conditions required to detect the correlation between transmitted and reflected speckle patterns and report the first experimental proof of its existence. Furthermore, we show that this correlation is robust and we provide a complete understanding of all its complex features, for scattering materials with thickness $L$ and scattering mean free path $\ell$ covering the entire range from single scattering $(L \lesssim \ell)$ to diffusive transport $(L \gg \ell)$. The data are supported by $3 \mathrm{D}$ numerical simulations and by a theoretical analysis of the line shape of the correlation function, as well as its dependence on the experimental parameters. The experiments and the theory embrace the complexity and the richness of the phenomenon, thus opening the way to its use as a basic ingredient in the design of new approaches for sensing, imaging, or communicating through opaque scattering media.

Although major properties of speckle patterns are well captured by modeling wave propagation with Gaussian random processes [22], we will show that the cross-sample correlation emerges from a non-Gaussian correction to the speckle properties. As a consequence, the correlation function is always small in amplitude but long range. In particular, it does not contain any feature oscillating at the wavelength scale. In addition, we will demonstrate that the correlation function is independent of the disorder strength in the regime of large optical thickness in which the medium is opaque. This means that changing the scattering mean free path $\ell$, for example, by changing the density of scatterers, does not affect the correlation function. Hence, the dimensionless conductance (proportional to $\ell$ ) is not the crucial parameter governing the cross-sample correlation measured here, contrary to the behavior of long-range correlations measured in transmission, which have been extensively studied $[7,8]$. Furthermore, we will show that the information content of the correlation function between reflection and transmission crucially depends on the distance to the sample. This is also in sharp contrast with long-range transmission correlations for which far-field and surface correlations are essentially related by Fourier transforms so that they carry the same information. Finally, we will identify a regime of moderate optical thickness, where the correlation function becomes anisotropic and keeps a memory of the illumination angle. This memory effect, due to long-range correlations, has never been detected before and is fundamentally different from the well-known memory effect observed in transmission or reflection and resulting from Gaussian statistics [15].

\section{MEASUREMENT OF THE TRANSMISSION- REFLECTION CORRELATION}

The experimental apparatus is shown in Fig. 1(a). A monochromatic wave (2-mW He-Ne laser) is incident at an angle of approximately $45^{\circ}$ on a suspension of $\mathrm{TiO}_{2}$ (a)

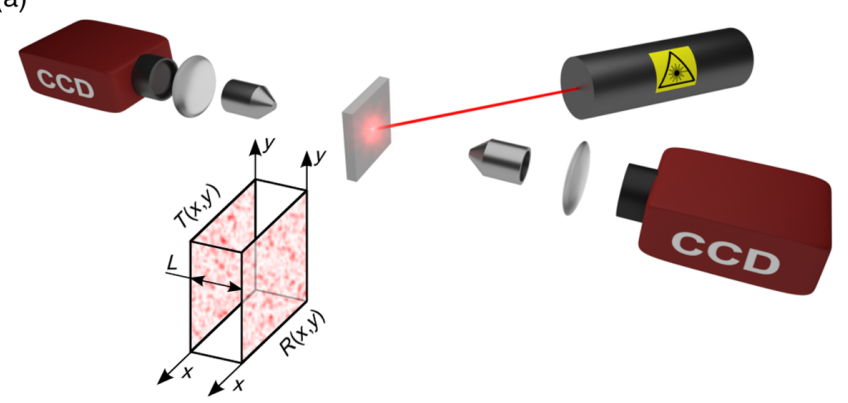

(b)
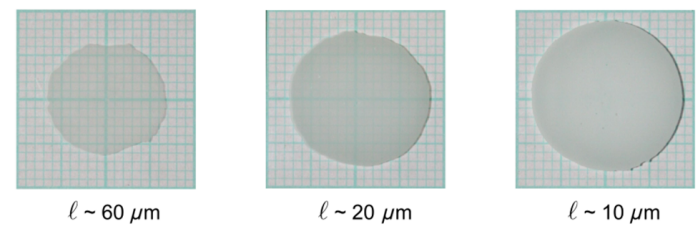

FIG. 1. (a) Experimental setup. A scattering slab, formed by a suspension of $\mathrm{TiO}_{2}$ particles in glycerol, is illuminated by a laser beam incident at an angle of approximately $45^{\circ}$. The speckle patterns on the two surfaces, $T(x, y)$ and $R(x, y)$ are recorded with two identical imaging systems. (b) Examples of samples with thickness $L=20 \mu \mathrm{m}$ but different $\mathrm{TiO}_{2}$ concentrations: from left to right, $5 \mathrm{~g} / \mathrm{dm}^{3}, 10 \mathrm{~g} / \mathrm{dm}^{3}$, and $40 \mathrm{~g} / \mathrm{dm}^{3}$, which correspond to a mean free path of $(60,20.4$, and 9.8) $\pm 2.5 \mu \mathrm{m}$, respectively.

particles in glycerol, held between two microscope slides to form a scattering slab. The slab thickness $L$ is controlled using calibrated spacers, and the mean free path $\ell$ is controlled by varying the $\mathrm{TiO}_{2}$ concentration (see Appendix A). Typical samples with different optical thickness $b=L / \ell$, from semitransparent to fully opaque, are shown in Fig. 1(b). For a set of given $L$ and $\ell$, we record the intensity patterns $R(\mathbf{r})$ and $T(\mathbf{r})$ on the surface of the sample in reflection and transmission, respectively, with two identical imaging systems, each composed of a $10 \times$ microscope objective, a planoconvex $150-\mathrm{mm}$ lens, and a CCD camera (Allied Vision Manta G-146). As the samples are liquid, the resulting speckle patterns change in time due to the Brownian motion of the scatterers, with a decorrelation time $\tau$ that depends strongly on the sample thickness. Choosing an integration time $<\tau$, and a time interval between successive measurements $>\tau$, allows us to measure speckle images $R(\mathbf{r})$ and $T(\mathbf{r})$ for a large ensemble of configurations of the disordered medium. For all our experiments, the integration time was set to $1 \mathrm{~ms}$. An example pair of images measured for a given realization of disorder is shown in Figs. 2(a) and 2(b). For each pair of $R$ and $T$, we calculate the correlation function $C^{R T}$, defined as

$$
C^{R T}(\Delta \mathbf{r})=\frac{\langle\delta R(\mathbf{r}) \delta T(\mathbf{r}+\Delta \mathbf{r})\rangle}{\langle R(\mathbf{r})\rangle\langle T(\mathbf{r}+\Delta \mathbf{r})\rangle},
$$


(a)

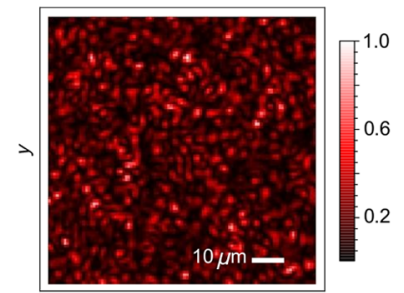

(c)

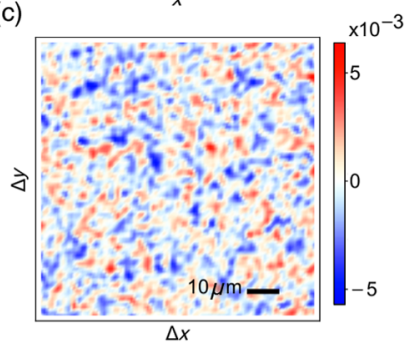

(b)

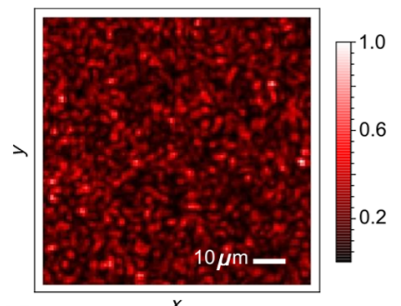

(d)

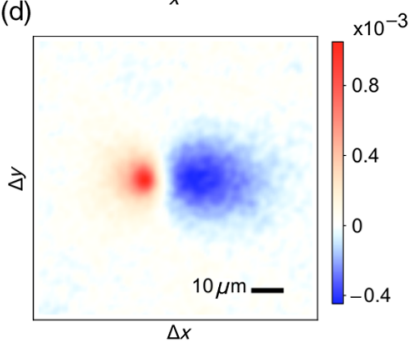

FIG. 2. Typical measured speckle patterns in transmission (a) and reflection (b), for a sample with $L=20 \mu \mathrm{m}$ and $\ell \simeq 20 \mu \mathrm{m}$. (c) Cross-correlation product between the speckle patterns in (a) and (b). (d) Correlation function $C^{R T}(\Delta x, \Delta y)$ obtained after additional ensemble averaging from $10^{4}$ realizations of the disorder. The long-range character of the correlation function, which extends far beyond the size of a speckle spot, is clearly visible.

where $\Delta \mathbf{r}=(\Delta x, \Delta y)$ is a transverse shift between the images, and $\delta f=f-\langle f\rangle$ denotes the statistical fluctuation of a random variable $f$, with $\langle\cdot\rangle$ the ensemble average. In the experiment, the averaging process is performed in two steps (see Appendix B). First, a cross-correlation product between $\delta R$ and $\delta T$, i.e., the integral $\int \delta R(\mathbf{r}) \delta T(\mathbf{r}+\Delta \mathbf{r}) d \mathbf{r}$, is taken for each realization of disorder. Plotted as a $2 \mathrm{D}$ map, the correlation product appears random, with a granularity similar to that of a speckle image [Fig. 2(c)]. Second, an ensemble averaging over the realizations of the disorder is taken, resulting in the appearance of a clear pattern in $C^{R T}(\Delta x, \Delta y)$ [Fig. 2(d)], and demonstrating that the transmitted and reflected speckle patterns are indeed correlated.

Speckle correlations are commonly divided into three categories: short-range correlations $\left(C_{1}\right)$ that decay with the separation between the observation points on the scale of the wavelength, long-range correlations $\left(C_{2}\right)$ that have a polynomial decay, and infinite-range correlations $\left(C_{3}\right)[8,9]$. The short-range correlation $C_{1}$ corresponds to the approximation of a field obeying Gaussian statistics [2], while $C_{2}$ and $C_{3}$ are non-Gaussian corrections. An additional infinite-range correlation $\left(C_{0}\right)$ appears under illumination by a point source located inside the medium [23]. One can see in Fig. 2(d) that the line shape of $C^{R T}(\Delta \mathbf{r})$ is much wider than a speckle spot, indicating that the dominant contribution to this correlation is long range in nature.

In order to characterize the line shape of the correlation function, and to probe its dependence on the sample

parameters, we measured $C^{R T}(\Delta \mathbf{r})$ for different values of $\ell$ and $L$, covering the full range from the single scattering $(L \lesssim \ell)$ to the multiple scattering $(L \gg \ell)$ regime. The results are summarized in Fig. 3 (center and right columns), where both 2D maps $C^{R T}(\Delta x, \Delta y)$ and cross sections along the line $\Delta y=0$ (indicated as a dotted line in the 2D maps) are displayed. It is interesting to note that both the shape and the sign of the reflection-transmission correlation substantially depend on $L$ and $\ell$. In the single scattering regime (optical thickness $b \lesssim 1$ ), $C^{R T}$ is dominated by a narrow peak (still much larger than a single speckle spot) with a negative side lobe. In the multiple scattering regime $(b \gg 1)$, $C^{R T}$ is dominated by a wide negative dip.

The short-range contributions to $C^{R T}\left(C_{1}\right)$ decay on the scale of the wavelength [2] and are negligible in all measurements since, in the reflection-transmission geometry, the observation points are separated by a distance $\sqrt{L^{2}+|\Delta \mathbf{r}|^{2}}$, which is much larger than $\lambda$ for even the thinnest sample (see Appendix $\mathrm{C}$ for a detailed discussion). Hence, $C^{R T}$ is necessarily a long-range correlation of the $C_{2}$ type. It is interesting to note that the reflection-transmission geometry naturally favors the observation of long-range correlations, without requiring any postprocessing to remove the $C_{1}$ contribution that dominates in the pure transmission geometry $[10,11,24,25]$. Another feature of the experiment is the illumination/detection geometry that excludes any contribution from specularly reflected and transmitted fields. Indeed, in the geometry in Fig. 1(a), the detectors do not collect the specularly reflected and transmitted averaged fields, but only the scattered light, i.e., the intensities of the fluctuating fields $T(\mathbf{r})=\left|\delta E_{T}(\mathbf{r})\right|^{2}$ and $R(\mathbf{r})=\left|\delta E_{R}(\mathbf{r})\right|^{2}$. This permits us to track various longrange correlations all the way from $b \lesssim 1$ to $b \gg 1$ by avoiding spurious interference terms in the intensity correlation function that would not be negligible in the single scattering regime. The contribution of the averaged fields to the full field correlation function is discussed in Appendix D.

\section{NUMERICAL SIMULATIONS}

To support the experimental data, we have performed full numerical simulations of wave propagation in threedimensional disordered media. In the simulations, the samples consist of slabs of dipole scatterers with random positions. The scalar wave equation is solved numerically using the coupled-dipole method [26]. Since the measurements are not resolved in polarization, and since the input light is expected to depolarize on a length scale on the order of $\ell$ [27], we neglect polarization and numerically solve the scalar wave equation. To limit the number of scatterers and save computational time, the polarizability $\alpha$ of each scatterer has been chosen to maximize the scattering cross section $\sigma_{s}=k^{4}|\alpha|^{2} / 4 \pi$, leading to $\alpha=4 i \pi / k^{3}$, where $k$ is the wave number. Adjusting the number density of 

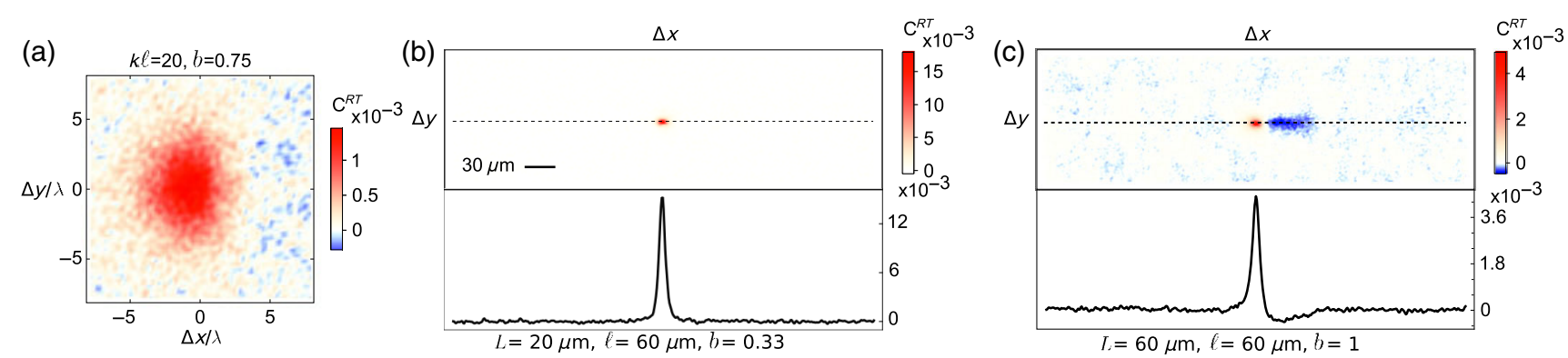

(d)

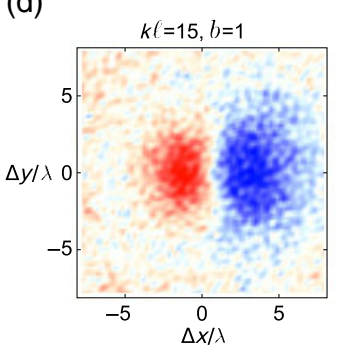

(e)
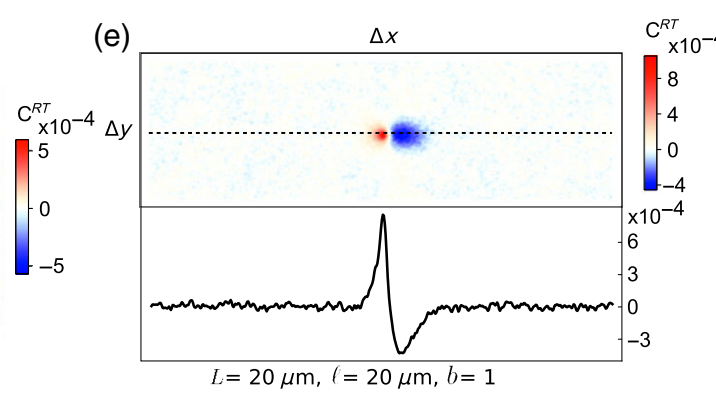

(g)
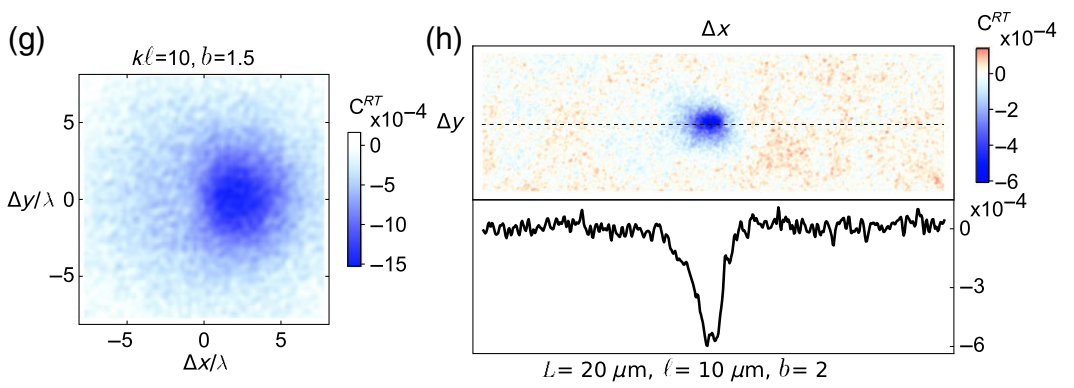
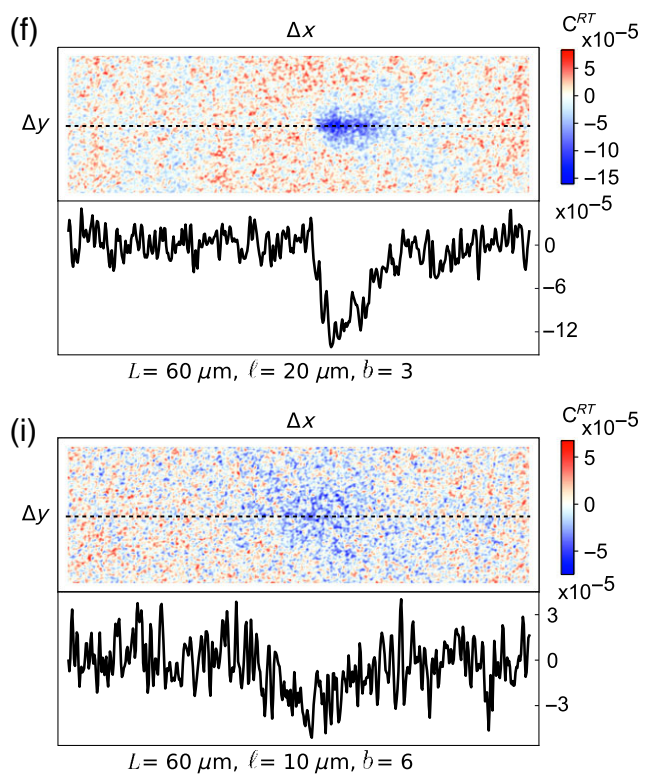

FIG. 3. Average reflection-transmission correlation function $C^{R T}$ for different values of $L$ and $\ell$ and the optical thickness $b=L / \ell$. Left column: 3D numerical simulations of 2D maps of $C^{R T}(\Delta x, \Delta y)$. Center and right columns: Experimental results. For clarity, both 2D maps of $C^{R T}(\Delta x, \Delta y)$ and cross sections along the line $\Delta y=0$ (indicated as a dotted line in the 2D maps) are displayed. Two regimes are identified. For moderate optical thickness $(b \lesssim 1)$, the correlation function is dominated by a narrow peak with a negative side lobe. For large optical thicknesses $(b>1)$, the correlation function is dominated by a wide negative dip.

scatterers $\rho$, we can vary the scattering mean free path $\ell=1 / \rho \sigma_{s}$ and simulate different kinds of samples. Solving numerically the coupled-dipole equations, we compute the scattered field at any point on the input and exit surfaces of the slab and deduce the correlation function $C^{R T}(\Delta \mathbf{r})$. The ensemble averaging is performed by computing the field for $N$ realizations of the positions of the scatterers. $N$ should be sufficiently large to satisfy $C^{R T} \gg \sigma / \sqrt{N}$, where $\sigma$ is the standard deviation of the unaveraged correlation function. In the multiple scattering regime, where $C^{R T} \sim$ $1 /(k L)^{2}$ (see below) and $\sigma \sim 1$, we get $N \gg(k L)^{4}$. As an example, for $k \ell=10$ and $b=1.5$, we have used 2685 dipoles and $N=2.6 \times 10^{7}$ configurations. The results of the simulations are displayed in Fig. 3 (left column) and are in very good agreement with the experimental data. The general shape of the correlation in the regimes $b<1, b \simeq 1$, and $b>1$ is well reproduced in the simulations. It is also worth noting that, in the experiment, $N$ must be replaced by the effective number of realizations $N_{\text {eff }}=N \mathcal{A} L^{-2} \gg N$ that takes into account the spatial averaging, with $\mathcal{A}$ the integration area in the speckle images. Taking advantage of the small decorrelation time of the medium and the large field of view of the camera, this effective number can be made large at will, allowing us to probe the reflectiontransmission correlation for sample thicknesses inaccessible in the simulations.

\section{THEORETICAL ANALYSIS}

In order to refine the analysis, and to get more physical insight, we have also used a formal perturbation theory, in which the correlation function $C^{R T}(\Delta \mathbf{r})$ defined in Eq. (1) is directly computed from a statistical ensemble averaging, without going through the intermediate crosscorrelation product used for the experimental data. Both averaging processes coincide provided that $\ell \gg \lambda$, a condition that is always satisfied in our experiments. Formal perturbation theory uses $1 / k \ell$ as a small parameter and relies on a diagrammatic formalism that allows one to derive explicit expressions of intensity correlation functions [6-8]. In the reflection-transmission geometry, 
care must be taken to properly account for leading contributions $[16,28]$.

Let us first discuss the regime of large optical thickness $L \gg \ell$ corresponding to Figs. 3(f)-3(i). Strikingly, we observe in this regime that $C^{R T}(\Delta \mathbf{r})$ is negative, in agreement with the prediction in Ref. [18]. This means that, for every bright spot in reflection (transmission), the corresponding area in transmission (reflection) is more likely to be darker, and vice versa. This feature is consistent with flux conservation arguments. Indeed, defining $T \propto$ $\int T(\mathbf{r}) \mathrm{d} \mathbf{r}$ and $R \propto \int R(\mathbf{r}) \mathrm{d} \mathbf{r}$, energy conservation imposes $T+R=1$ for a nonabsorbing medium, from which it follows that $\int C^{R T}(\Delta \mathbf{r}) \mathrm{d} \Delta \mathbf{r} \propto\langle\delta T \delta R\rangle=-\left\langle\delta T^{2}\right\rangle<0$. Note that the existence of negative long-range $C_{2}$ correlations has been previously pointed out in Refs. [17,29].

We stress that the previous simple flux conservation argument imposes a global constraint on the correlation, but does not determine its sign for each position $\Delta \mathbf{r}$. Only a full theoretical calculation accounting for the interferences between crossing paths can give the full line shape of $C^{R T}(\Delta \mathbf{r})$. Nonetheless, an intuitive understanding of the local sign of the reflection-transmission speckle correlation can be built in the following way. In a thick scattering slab, interferences create large fluctuations of the wave intensity at the scale of the wavelength (bulk speckle pattern). These intensity fluctuations create local fluctuations of the energy flux that act as a source term for the diffusive transport of the intensity towards the medium boundaries. This mechanism is formally analogous to that of particle diffusion driven by Langevin forces in a molecular fluid [30,31]. Because of local flux conservation, a positive flux fluctuation pointing towards the transmission interface has to be compensated on average by a negative fluctuation pointing towards the reflection interface, giving rise to a local negative correlation. This analysis shows that the existence of R-T correlations does not rely on global energy conservation, so that we expect the correlation to be robust against absorption.

In Appendix E, we have refined the theoretical analysis performed in Ref. [18] in the regime $L \gtrsim \ell \gg \lambda$. We find that both the amplitude and the width of the correlation function depend on $L$ and $\ell$, as in the experimental data in Figs. 3(f)-3(i). For $L \gg \ell$, the dominant diagrams belong to the class represented in Fig. 4(a), which are typical of long-range $C_{2}$ correlations. They predict a correlation function that is isotropic, independent of the angle of incidence, and scales as $C^{R T}(\Delta \mathbf{r})=C_{2}^{R T}(\Delta \mathbf{r})=$ $-f(|\Delta \mathbf{r}|) /(k L)^{2}$, where $f$ is a dimensionless function that decays on a range $|\Delta \mathbf{r}| \simeq L[18,32]$. This long-range character of the correlation function originates from the crossing of two diffusive paths that probe a transverse distance $L$ before escaping, as represented in Fig. 4(a). Note that this path crossing is the analog of the source term in the Langevin picture mentioned above. Moreover, the correlation function in this regime is independent of the disorder strength $k \ell$, which makes it strikingly different

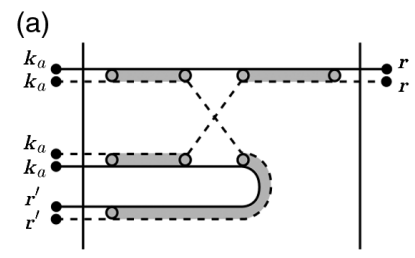

(b)

FIG. 4. Diagrams contributing to the $C^{R T}(\Delta \mathbf{r})$ correlation. An intensity correlation depends on two intensities (four fields) that propagate through the sample, therefore involving four inputs and four outputs. Shaded tubes represent diffusive paths and open circles stand for scatterers; single solid lines stand for averaged fields and single dashed lines for their complex conjugates. The diagram in panel (a) is representative of the class of $C_{2}$ diagrams describing the negative contribution of the correlation function at large optical thickness $(L \gg \ell)$. Panel (b) represents the class of $C_{0}$-type diagrams that contribute to the positive peak dominant in the regime $\ell \sim L \gg \lambda$.

from that observed in a pure transmission geometry, for which $C_{2}^{T T} \sim 1 /[(k \ell)(k L)] \propto 1 / g$, where $g$ is the dimensionless conductance of the sample [9]. Another important difference between $C_{2}^{R T}$ and $C_{2}^{T T}$ is the evolution of their information content with respect to the detection scheme. Although $C_{2}^{T T}$ contains the same information whether it is measured on the sample surface or in the far field, this is not the case for $C_{2}^{R T}$. Indeed, in the far field, we have $C^{R T}\left(\mathbf{k}_{b}, \mathbf{k}_{b^{\prime}}\right) \sim \int C^{R T}(\Delta \mathbf{r}) \mathrm{d} \Delta \mathbf{r}=$ const for any pair of observation directions $\mathbf{k}_{b}, \mathbf{k}_{b^{\prime}}$, as the information content is spread uniformly over all degrees of freedom. We give a more detailed explanation of this phenomenon in Appendix E. For this reason, we focus our discussion on the correlations measured on the sample surface.

In the regime of moderate optical thickness $\ell \sim L \gg \lambda$, where single scattering is expected to dominate, an intensity correlation extending far beyond the size of a single speckle spot is still observed [see Figs. 3(c)-3(e)], but with a positive peak appearing in the vicinity of the negative contribution. The apparent relative position and amplitude between the peak and the dip depends on the angle of incidence of the illumination (see Appendix F). Contrary to the negative dip in the correlation function observed at large optical thickness, the line shape is anisotropic, with negative side lobes (hardly visible in the experimental data in Figs. 3(c)-3(e), but visible in the calculations presented in Appendix F) that are more pronounced along the direction of the projection of the incident beam onto the sample surface. Moreover, the amplitudes of both the positive peak and the side lobes substantially depend on the incidence angle. These two features of the correlation function (long-range extent and dependence on the angle of incidence) suggest a qualitative description based on diagrams of the class represented in Fig. 4(b). Such diagrams satisfy both properties simultaneously. The field exchange, which creates the correlation, occurs in the first scattering event and encodes a phase difference in the 
subsequent diffusive paths (shaded tubes) that depends on the angle of incidence. Moreover, the diffusive propagation provides the long-range behavior. The theoretical evaluation of these diagrams is detailed in Appendix G. They lead to a contribution to the correlation function scaling as $1 /(k L)^{4}$ for $b \gg 1$. This is consistent with the fact that, according to the measurements and the numerical simulations, this contribution has to be negligible at large optical thickness, where the $C_{2}^{R T}$ correlation function discussed previously scaling as $1 /(k L)^{2}$ dominates. The observed anisotropy in the correlation function is also well reproduced, supporting the relevance of the analysis based on the diagrams in Fig. 4(b). Interestingly, these diagrams are formally similar to those leading to the infinite-range correlations $C_{0}$ observed when the sample is excited by a point source $[23,33]$. In a nonabsorbing medium, as a consequence of energy conservation, the $C_{0}$ contribution is related to the fluctuations of the local density of states at the source position $[34,35]$. In the present context, where a plane wave excitation is used, the $C_{0}$-type contribution to the reflection-transmission correlation function is long range and satisfies $\int\left\langle C_{0}^{R T}(\Delta \mathbf{r})\right\rangle \mathrm{d} \Delta \mathbf{r}=$ 0 . This property also leads to the conclusion that the $C_{0}$-type contribution is specific to speckle patterns measured on the surface of the sample and vanishes in the case of far-field angular measurements (see Appendix G).

Finally, in the quasiballistic regime $\ell \gg L \gg \lambda$, which is not the focus of our experiment, we expect the correlation $C^{R T}$ to contain additional contributions to $C_{2}$ and $C_{0}$ (see the discussion in Appendix G), which still result in an overall positive peak. Note that this positive correlation does not contradict the flux conservation argument mentioned earlier. Indeed, this argument rigorously applies for intensity correlations built from the total fields (including the averaged reflected and transmitted fields), which coincides with the measured correlation Eq. (1) at large optical thickness only (see Appendix D).

\section{CONCLUSIONS}

In summary, we have demonstrated experimentally the existence of a cross-correlation between the speckle patterns measured in reflection and transmission on the surface of a disordered medium. The correlation persists in the regime of large optical thickness $L \gg \ell$, in which the sample is opaque due to multiple scattering. The measurements are supported by 3D numerical simulations and have been analyzed using a perturbative theory (valid when $\ell \gg \lambda$ ). We have found that the reflection-transmission correlation has two contributions: a positive peak dominant at moderate optical thicknesses $L \lesssim \ell$ and a negative dip dominant in the multiple scattering regime $L \gg \ell$, which we interpret as (respectively) $C_{0^{-}}$and $C_{2}$-type scattering sequences. In the regime $L \gg \ell$, the amplitude of $C^{R T}$ scales as $1 /(k L)^{2}$ in $3 \mathrm{D}$, but, at the same time, the range over which the correlation has an effect grows as $L^{2}$, compensating the decrease in amplitude with the increase of the number of speckle spots contributing to the cross information [32]. The possibility to extract information on the transmitted speckle from a measurement limited to the reflection half-space offers new possibilities for the detection of objects hidden behind opaque scattering media, including ghost imaging schemes, and for the control of wave propagation by wavefront shaping techniques [36,37].

The research data supporting this publication are openly available from the University of Exeter's institutional repository [38].

\section{ACKNOWLEDGMENTS}

This work was supported by the Leverhulme Trust's Philip Leverhulme Prize, and by LABEX WIFI (Laboratory of Excellence within the French Program "Investments for the Future") under references ANR-10-LABX-24 and ANR-10IDEX-0001-02 PSL*. I. S. and A. M. P.-D. acknowledge support from EPSRC (EP/L015331/1) through the Centre of Doctoral Training in Metamaterials (XM2). N. F. acknowledges financial support from the French "Direction Générale de l'Armement" (DGA).

\section{S., A. M. P., N. F. are contributed equally.}

\section{APPENDIX A: MEAN FREE PATH CHARACTERIZATION}

In order to determine the mean free path of the three samples with different concentrations of the $\mathrm{TiO}_{2}$, we used the well-known Lambert-Beer law $I=I_{0} e^{-L / \ell_{e}}$, where $I$ and $I_{0}$ are the transmitted and initial intensities, respectively; $L$ is the thickness of the sample; and $\ell_{e}$ the extinction length. This law measures the attenuation of

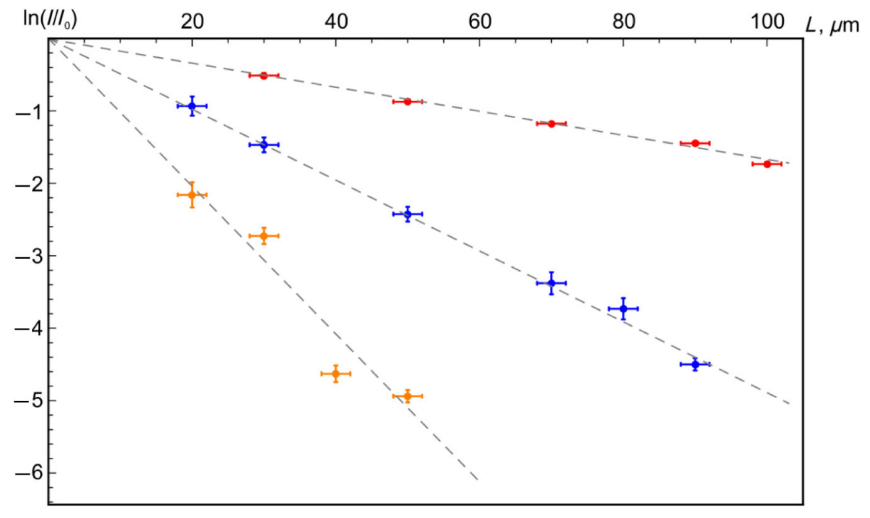

FIG. 5. Experimental data to determine the mean free path of the three different sample concentrations we used. In red are the data corresponding to the concentration of $50 \mathrm{mg}$ of $\mathrm{TiO}_{2}$ in $10 \mathrm{~mL}$ of glycerol, in blue the concentration of $150 \mathrm{mg}$ of $\mathrm{TiO}_{2}$ in $10 \mathrm{~mL}$ of glycerol, and in yellow the data corresponding to the concentration of $400 \mathrm{mg}$ of $\mathrm{TiO}_{2}$ in $10 \mathrm{ml}$ of glycerol. The black dashed lines represent the Lambert-Beer law fitting to the corresponding experimental data. 
the ballistic light when going through the sample. Since absorption in the sample of $\mathrm{TiO}_{2}$ and glycerol is negligible (albeit nonzero) compared to scattering, we can consider $\ell_{e} \approx \ell$. We measured the attenuation of the ballistic beam for different thicknesses of the sample and obtained the scattering mean free path of the three different samples by fitting the Lambert-Beer law, as shown in Fig. 5.

The scattering mean free paths for the samples with concentrations of $50 \mathrm{mg}$ of $\mathrm{TiO}_{2}, 150 \mathrm{mg}$ of $\mathrm{TiO}_{2}$, and $400 \mathrm{mg}$ of $\mathrm{TiO}_{2}$ in $10 \mathrm{ml}$ of glycerol were found to be, respectively, $58.5 \pm 1.3 \mu \mathrm{m}, 18.0 \pm 0.5 \mu \mathrm{m}$, and $6.9 \pm 0.7 \mu \mathrm{m}$.

\section{APPENDIX B: EXPERIMENTAL DETERMINATION OF THE CORRELATION FUNCTION}

Experimentally, the determination of the intensity correlation function $C^{R T}(\Delta \mathbf{r})$ defined by Eq. (1) is performed in two steps. First, a spatially averaged function $\overline{C^{R T}}(\Delta \mathbf{r}) \propto \int \delta R(\mathbf{r}) \delta T(\mathbf{r}+\Delta \mathbf{r}) d \mathbf{r}$ is calculated for each pair of reflected and transmitted speckle images. Then, an ensemble averaging is performed, leading to the correlation function $\left\langle\overline{C^{R T}}(\Delta \mathbf{r})\right\rangle$. In this appendix, we comment on the definition of $\overline{C^{R T}}(\Delta \mathbf{r})$ that allows us to remove some experimental artifacts, and we detail the necessary conditions for the correlation function $\left\langle\overline{C^{R T}}(\Delta \mathbf{r})\right\rangle$ to coincide with the usual correlation function $C^{R T}(\Delta \mathbf{r})$ that only involves ensemble averaging.

Measuring images (including speckle patterns) with coherent light inevitably leads to artifacts due to interference of the signal with scattered light. Figures 6(a) and 6(b) show an example of a raw measurement for the speckle patterns in transmission and reflection. From a single measurement, it is almost impossible to notice, but all the raw measurements sit on an irregular fringe pattern background due to the reflections in the protective glass window in front of the CCD detector. In fact, if we average the raw measurements over disorder, the speckle patterns average out, and the inhomogeneous background becomes apparent [see Figs. 6(c) and 6(d)].

If one were to use the raw measurements to find the cross-correlation function, the correlation between the inhomogeneous background patterns would dominate and obscure any real speckle correlation [see Fig. 6(e)]. These artifacts can be eliminated using the following procedure. Let us denote by $S_{m}$ the measured speckle pattern in reflection or transmission, and by $F$ the unwanted fringes. We have $S_{m}=S+F$, where $S$ is the desired speckle pattern, and $F$ is unknown but is also the same for each realization of disorder. Thus, we can calculate $S_{m}-\left\langle S_{m}\right\rangle=S+F-(\langle S\rangle+F)=S-\langle S\rangle=\delta S$. This procedure eliminates $F$ and directly leads to a measurement of $\delta S$. For this reason, we have defined a spatially averaged function
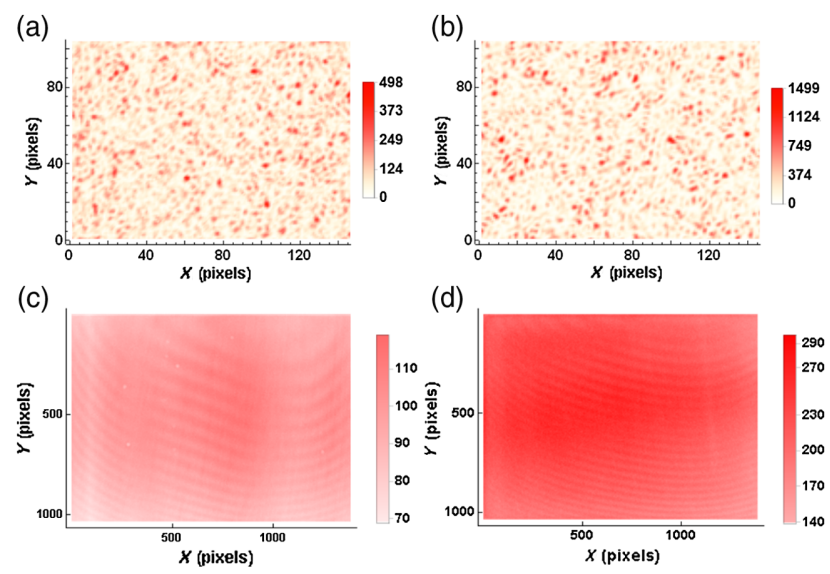

(d)
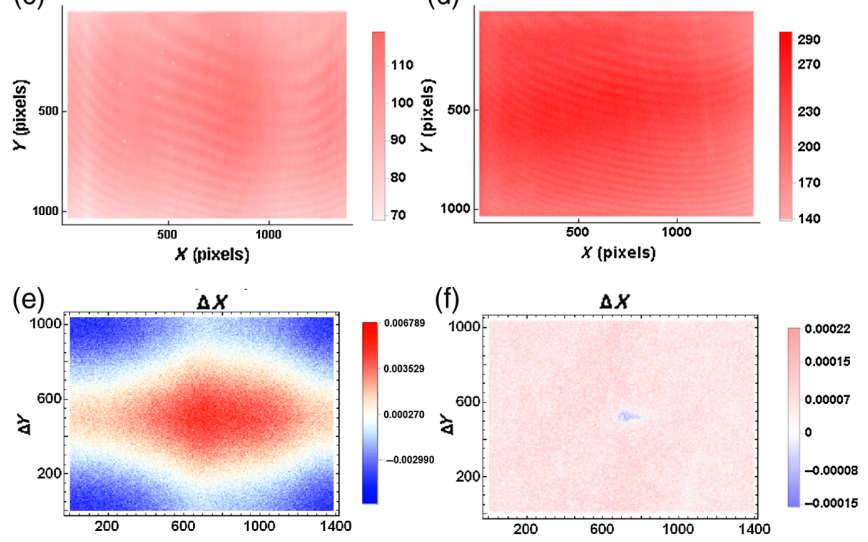

FIG. 6. Typical raw average intensity measurement in reflection (a) and transmission (b), with the averaged intensity distribution over 2000 realizations of disorder in reflection (c) and transmission (d). The inhomogeneous background is clearly visible in both $\langle R\rangle$ and $\langle T\rangle$. (e) Direct cross-correlation of $R$ and $T$ (without subtracting the background). (f) Cross-correlation between $\delta R$ and $\delta T$ as described in the main text.

$$
\overline{C^{R T}}(\Delta \mathbf{r})=\mathcal{N}_{1}^{-1} \overline{\delta R(\mathbf{r}) \delta T(\mathbf{r}+\Delta \mathbf{r})},
$$

with the normalization factor

$\mathcal{N}_{1}={\overline{\left[\delta R(\mathbf{r})-\overline{\delta R(\mathbf{r})}^{2}\right.}}^{1 / 2} \times \overline{[\delta T(\mathbf{r}+\Delta \mathbf{r})-\overline{\delta T(\mathbf{r}+\Delta \mathbf{r})}}^{2}{ }^{1 / 2}$.

Here, the overline represents the spatial average over the coordinate $\mathbf{r}$. This quantity is directly accessible from the experimental data and is free of artifacts, as shown in Fig. 6(f).

In the experiment, the spatially averaged function $\overline{C^{R T}}(\Delta \mathbf{r})$ is averaged over an ensemble of realizations of disorder, leading to a correlation function $\left\langle\overline{C^{R T}}(\Delta \mathbf{r})\right\rangle$. Assuming that spatial and ensemble averaging are equivalent, we can write $\left\langle\overline{C^{R T}}(\Delta \mathbf{r})\right\rangle \simeq \mathcal{N}_{2}^{-1}\langle\delta R(\mathbf{r}) \delta T(\mathbf{r}+\Delta \mathbf{r})\rangle$, with

$$
\begin{aligned}
\mathcal{N}_{2}= & \left\langle[\delta R(\mathbf{r})-\langle\delta R(\mathbf{r})\rangle]^{2}\right\rangle^{1 / 2} \\
& \times\left\langle[\delta T(\mathbf{r}+\Delta \mathbf{r})-\langle\delta T(\mathbf{r}+\Delta \mathbf{r})\rangle]^{2}\right\rangle^{1 / 2}
\end{aligned}
$$

This correlation differs from Eq. (1), since the normalization factor $\mathcal{N}_{2}$ involves intensity fluctuations in reflection and transmission, and not averaged values. Since the experiments are carried out in the weak scattering regime 


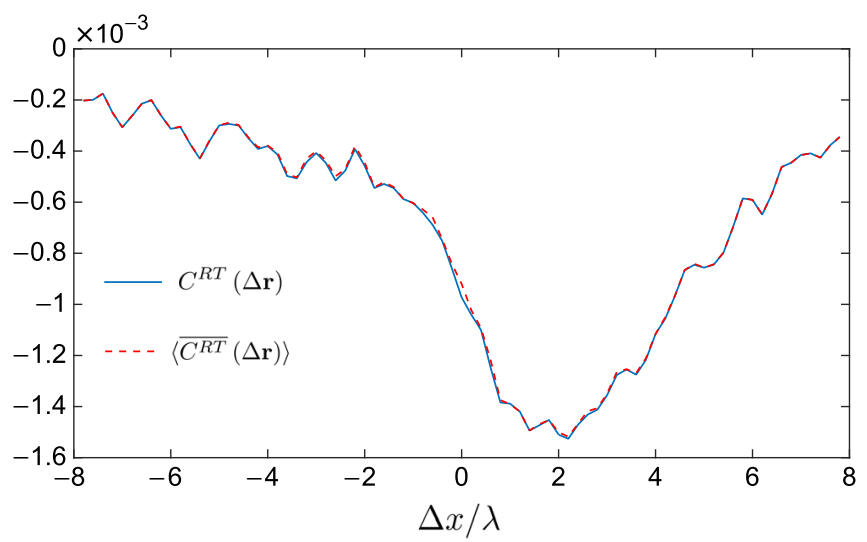

FIG. 7. Comparison between the two correlation functions $\left\langle\overline{C^{R T}}(\Delta \mathbf{r})\right\rangle$ and $C^{R T}(\Delta \mathbf{r})$. The transverse distance $\Delta r=\Delta x$ is varied along the direction of the illumination plane. The parameters of the $3 \mathrm{D}$ numerical simulation are $L / \ell=1.5$, $k \ell=10, \theta_{a} \simeq 45^{\circ}$.

$k \ell \gg 1$, we can assume that the intensity in each speckle pattern follows a Rayleigh statistics to a very good approximation. This amounts to neglecting non-Gaussian contributions to the field in each speckle pattern. In this case, we have $\left\langle\delta R^{2}\right\rangle=\langle R\rangle^{2}$ and $\left\langle\delta T^{2}\right\rangle=\langle T\rangle^{2}$, and

$$
\left\langle\overline{C^{R T}}(\Delta \mathbf{r})\right\rangle \simeq C^{R T}(\Delta \mathbf{r})=\frac{\langle\delta R(\mathbf{r}) \delta T(\mathbf{r}+\Delta \mathbf{r})\rangle}{\langle R(\mathbf{r})\rangle\langle T(\mathbf{r}+\Delta \mathbf{r})\rangle} .
$$

We illustrate the good agreement between $\left\langle\overline{C^{R T}}(\Delta \mathbf{r})\right\rangle$ and $C^{R T}(\Delta \mathbf{r})$ in Fig. 7, where both correlations have been calculated numerically for a $3 \mathrm{D}$ scattering medium with optical thickness $b=1.5$ and scattering strength $k \ell=10$.

\section{APPENDIX C: GAUSSIAN AND NON-GAUSSIAN CONTRIBUTIONS TO THE CORRELATION FUNCTION}

The calculation of the correlation function given in Eq. (1) requires us to evaluate $\left\langle R(\mathbf{r}) T\left(\boldsymbol{r}^{\prime}\right)\right\rangle=\left\langle\delta E_{R}(\mathbf{r}) \delta E_{R}(\mathbf{r})^{*} \delta E_{T}\left(\mathbf{r}^{\prime}\right) \delta E_{T}\left(\mathbf{r}^{\prime}\right)^{*}\right\rangle$,

where $\mathbf{r}^{\prime}=\mathbf{r}+\Delta \mathbf{r}$. The Gaussian contribution, usually denoted $C_{1}$, is obtained by pairing fields to form averages of complex conjugate pairs. On the other hand, nonGaussian contributions necessarily involve scattering paths that connect four fields, since $\left\langle\delta E_{R}(\mathbf{r})\right\rangle=\left\langle\delta E_{T}(\mathbf{r})\right\rangle=0$. By noting $\langle\ldots\rangle_{c}$ as the non-Gaussian contributions, we obtain

$$
\begin{aligned}
C^{R T}(\Delta \mathbf{r})= & \frac{\left|\left\langle\delta E_{R}(\mathbf{r}) \delta E_{T}\left(\mathbf{r}^{\prime}\right)^{*}\right\rangle\right|^{2}}{\langle R(\mathbf{r})\rangle\left\langle T\left(\mathbf{r}^{\prime}\right)\right\rangle} \\
& +\frac{\left\langle\delta E_{R}(\mathbf{r}) \delta E_{R}(\mathbf{r})^{*} \delta E_{T}\left(\mathbf{r}^{\prime}\right) \delta E_{T}\left(\mathbf{r}^{\prime}\right)^{*}\right\rangle_{c}}{\langle R(\mathbf{r})\rangle\left\langle T\left(\boldsymbol{r}^{\prime}\right)\right\rangle} .
\end{aligned}
$$

The first term of Eq. (C2) is the $C_{1}$ contribution. This contribution can be large, of the order of unity, if the distance between observation points is smaller than the wavelength. This is always possible in the T-T configuration, where points $\mathbf{r}$ and $\mathbf{r}^{\prime}$ are measured in the same plane: The $C_{1}$ contribution dominates the correlation $C^{T T}(\Delta \mathbf{r})$ for $\Delta r \lesssim \lambda$. On the contrary, in the R-T configuration, the distance between observation points is $\sqrt{L^{2}+\Delta r^{2}}$. Therefore, the $C_{1}$ contribution is always negligible for $L \gg \lambda$, as is the case in our experiment. We have checked numerically that this statement remains valid at moderate optical thickness, where the diffusion approximation breaks down. In Fig. 8, we compare the full correlation $C^{R T}(\Delta \mathbf{r})$ with the $C_{1}$ contribution and the connected contribution [last term of Eq. (C2)], calculated from a microscopic wave propagation simulation in a three-dimensional slab of optical thickness $L / \ell=1$ and scattering strength $k \ell=15$. We observe that the full correlation [Fig. 8(a)] is well approximated by its connected part [Fig. 8(b)], indicating that the $C_{1}$ contribution [Fig. 8(c)] is indeed negligible. Other simulations (not shown) have revealed that the $C_{1}$ contribution becomes important for $k L \lesssim 1$, as expected. This analysis shows that the R-T configuration is particularly adapted to access and study non-Gaussian quantities
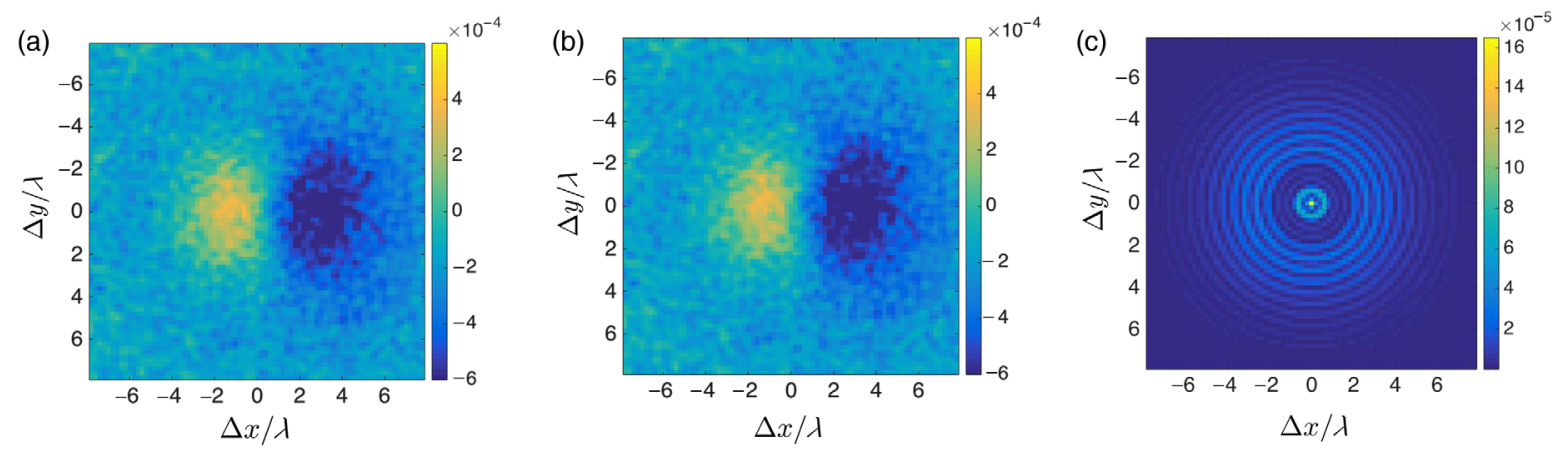

FIG. 8. Correlation function $C^{R T}$ calculated from numerical simulation of the wave equation in a 3D slab. (a) Full correlation. (b) Connected part of the correlation [second term of the rhs of Eq. (C2)]. (c) $C_{1}$ contribution of the correlation [first term of the rhs of Eq. (C2)]. The parameters are $k \ell=15, L / \ell=1, \theta_{a} \simeq 45^{\circ}$. 
in mesoscopic physics, such as the connected and longrange contributions $\left\langle\delta E_{R}(\mathbf{r}) \delta E_{R}(\mathbf{r})^{*} \delta E_{T}\left(\mathbf{r}^{\prime}\right) \delta E_{T}\left(\mathbf{r}^{\prime}\right)^{*}\right\rangle_{c}$. Indeed, the latter dominate the correlation in the full range from the deep diffusive $(L \gg \ell)$ to the quasiballistic $(L \lesssim \ell)$ regime. This is in sharp contrast with the T-T configuration, for which it is difficult to extract the small non-Gaussian part of the correlation: This requires a delicate fitting procedure or an additional field measurement that can be cumbersome with optical waves; see, for instance, Refs. [33,39].

\section{APPENDIX D: R-T CORRELATION BUILT FROM THE TOTAL REFLECTED AND TRANSMITTED FIELDS}

The experiment is performed with a laser illumination oriented with a nonzero angle with respect to the sample surface, which allows us to measure the intensity of the fluctuating part of the fields, $R(\mathbf{r})=\left|\delta E_{R}(\mathbf{r})\right|^{2}$ and $T\left(\mathbf{r}^{\prime}\right)=\left|\delta E_{T}\left(\mathbf{r}^{\prime}\right)\right|^{2}$, only. We want to discuss here what the R-T correlation would be if the mean fields were also measured. Let us note $\tilde{X}(\mathbf{r})=\left|E_{X}(\mathbf{r})\right|^{2}=\mid\left\langle E_{X}(\mathbf{r})\right\rangle+$ $\left.\delta E_{X}(\mathbf{r})\right|^{2}$, where $X$ stands for $R$ or $T$. By expressing $\tilde{X}$ in terms of $X$ and using $\langle\tilde{X}\rangle=\langle X\rangle+\left|\left\langle E_{X}\right\rangle\right|^{2}$, we get

$$
\begin{aligned}
\langle\delta \tilde{R}(\mathbf{r}) & \left.\delta \tilde{T}\left(\mathbf{r}^{\prime}\right)\right\rangle \\
= & \left\langle\delta R(\mathbf{r}) \delta T\left(\mathbf{r}^{\prime}\right)\right\rangle+2 \operatorname{Re}\left[\left\langle E_{T}\left(\mathbf{r}^{\prime}\right)\right\rangle\left\langle\delta E_{T}\left(\mathbf{r}^{\prime}\right)^{*} R(\mathbf{r})\right\rangle\right. \\
& +\left\langle E_{R}(\mathbf{r})\right\rangle\left\langle\delta E_{R}(\mathbf{r})^{*} T\left(\mathbf{r}^{\prime}\right)\right\rangle \\
& +\left\langle E_{R}(\mathbf{r})\right\rangle\left\langle E_{T}\left(\mathbf{r}^{\prime}\right)\right\rangle^{*}\left\langle\delta E_{R}(\mathbf{r})^{*} \delta E_{T}\left(\mathbf{r}^{\prime}\right)\right\rangle \\
& \left.+\left\langle E_{R}(\mathbf{r})\right\rangle\left\langle E_{T}\left(\mathbf{r}^{\prime}\right)\right\rangle\left\langle\delta E_{R}(\mathbf{r}) \delta E_{T}\left(\mathbf{r}^{\prime}\right)\right\rangle^{*}\right] .
\end{aligned}
$$

Hence, the R-T correlation built from the total reflected and transmitted fields contains additional interferences between the mean fields and the scattered fields. We illustrate their role in Fig. 9, where we compare $C^{R T}$ [panel (a)] and $\tilde{C}^{R T}$ [panel (b)] calculated for a 3D disordered slab $(L / \ell=1$,
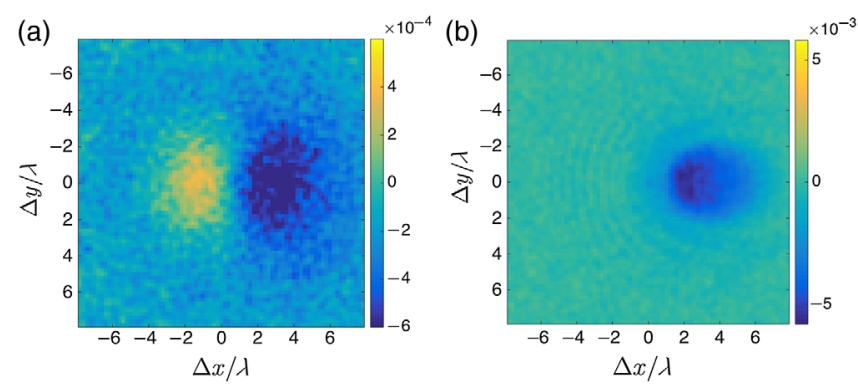

FIG. 9. Comparison of two R-T correlation functions calculated from numerical simulation of the wave equation in a $3 \mathrm{D}$ slab. (a) Correlation function $C^{R T}(\Delta \mathbf{r})=\langle\delta R(\mathbf{r}) \delta T(\mathbf{r}+\Delta \mathbf{r})\rangle /\langle R(\mathbf{r})\rangle$ $\langle T(\mathbf{r}+\Delta \mathbf{r})\rangle$ built from fluctuating parts of the fields. (b) Correlation function $\tilde{C}^{R T}=\langle\delta \tilde{R}(\mathbf{r}) \delta \tilde{T}(\mathbf{r}+\Delta \mathbf{r})\rangle /\langle\tilde{R}(\mathbf{r})\rangle\langle\tilde{T}(\mathbf{r}+\Delta \mathbf{r})\rangle$ built from the full fields (see text for details). The parameters are $k \ell=15, L / \ell=1, \theta_{a}=45^{\circ}$.
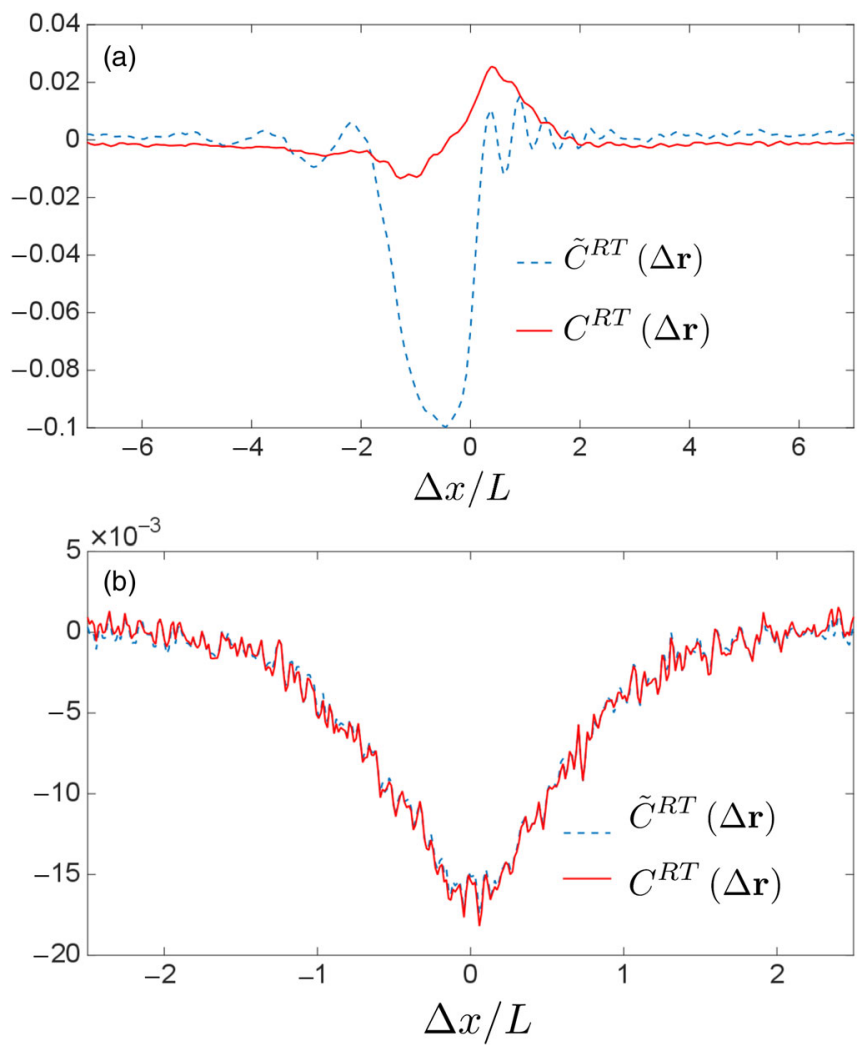

FIG. 10. Comparison of two R-T correlation functions calculated from numerical simulation of the wave equation in a $2 \mathrm{D}$ slab. (a) Moderate optical thickness $L=\ell$ and shifted incidence $\theta_{a} \simeq-35^{\circ}$. (b) Large optical thickness $L / \ell=7$ at normal incidence $\theta_{a}=0$. The other parameter is $k l=10$.

$k \ell=15$, and illumination angle $\theta_{a}=45^{\circ}$ ). On top of the long-range component of $C^{R T}, \tilde{C}^{R T}$ also exhibits tiny oscillating contributions and additional long-range contributions due to the four terms of Eq. (D1). These contributions, negligible in the deep diffusive regime $L \gg \ell$, become important at moderate optical thickness, $L \sim \ell$. This explains why the positive contribution to the longrange correlation discussed in this work was not detected in Ref. [18], where only $\tilde{C}^{R T}$ was analyzed. In Fig. 10, we compare both correlation functions, calculated from 2D numerical simulations such as those performed in Ref. [18], for two different optical depths. At large optical depth, the two correlations are equal, while it is no longer the case in the regime $L \sim \ell$, where interference terms dominate.

\section{APPENDIX E: ANALYTICAL CALCULATION OF $C_{2}^{R T}(\Delta r)$}

In this appendix, we refine the calculation of $C_{2}^{R T}$ that was previously performed by some of us in Ref. [18]. Let us first recall the physical picture that gives rise to the longrange $C_{2}$ correlation. The correlator (B4) is the average of four fields (measured at the two detector positions) that can be decomposed as sums over all scattering paths. The idea 
is to select paths that give a nonvanishing contribution to the average. The $C_{2}^{R T}$ contribution is obtained by considering pairs of fields that propagate diffusively in the disordered medium until their paths cross at an arbitrary position inside the medium. When this crossing occurs, pairs of fields exchange their partners to form new pairs that travel again diffusively through the system until they reach the output boundaries. There are two different ways to do so, as represented in Fig. 11. For the same reason that made $C_{1}^{R T}$ negligible (see Appendix $\mathrm{C}$ ), the diagram represented in Fig. 11(b) is negligible for all positions $\mathbf{r}$ and $\boldsymbol{r}^{\prime}$ in the regime $k L \gg 1$.

Let us stress here the difference between $C_{2}^{R T}$ and $C_{2}^{T T}$. $C_{2}^{T T}$ is made of the same diagrams as in Fig. 11, but with the point $\boldsymbol{r}^{\prime}$ in the transmission plane. As a result, the diagram of Fig. 11(b) is not negligible for observation points in the same transmission plane. In particular, this diagram with observation points in the far field is the Fourier transform of the one represented in Fig. 11(a), with points at the sample surface. Conversely, for $C_{2}^{R T}$, since the diagram of Fig. 11(b) is negligible, we measure in the far field the contribution of Fig. 11 (a) only,

$$
C^{R T}\left(\mathbf{k}_{b}, \mathbf{k}_{b}^{\prime}\right)=\int \frac{\mathrm{d} \Delta \mathbf{r}}{\mathcal{A}} C^{R T}(\Delta \mathbf{r}),
$$

for any couple of observation directions $\mathbf{k}_{b}, \mathbf{k}_{b}^{\prime}$. Here, $\mathcal{A}$ is the transverse area covered by the input illumination. Although $C_{2}^{T T}$ contains the same information whether it is measured at the sample surface or angularly in the far field, this is not the case for $C_{2}^{R T}$.

Mathematically, the contribution of Fig. 11(a) to the correlator $(\mathrm{C} 1)$ reads (a)

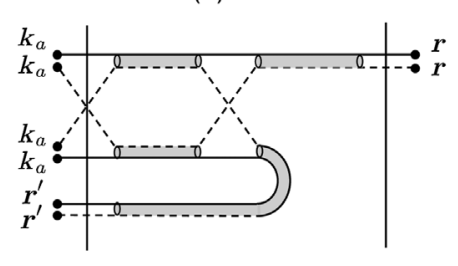

(b)

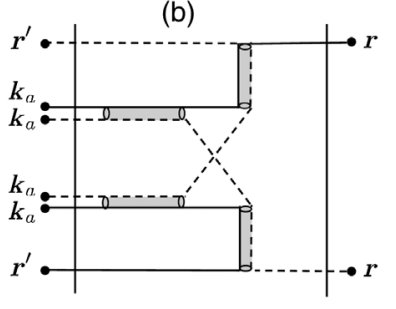

FIG. 11. Typical diagrams contributing to the connected fourfield correlations in R-T. Panel (a) corresponds to the case where the fields exchange at the entrance, propagate, and exchange inside the medium. After this exchange, they can travel diffusively through long distances to eventually be measured at the desired points. Panel (b) corresponds to the case where the two pairs of fields propagate first together, then exchange inside the medium, and, at the end, have to exchange again to eventually be measured at two different points. The output vertex of this diagram is identical to the output vertex of the $C_{1}$ correlation.

$$
\begin{aligned}
& \left\langle\delta E_{R}(\mathbf{r}) \delta E_{R}(\mathbf{r})^{*} \delta E_{T}\left(\mathbf{r}^{\prime}\right) \delta E_{T}\left(\mathbf{r}^{\prime}\right)^{*}\right\rangle_{C_{2}} \\
& =\int\left|\left\langle E\left(\mathbf{r}_{1}\right)\right\rangle\right|^{2}\left|\left\langle E\left(\mathbf{r}_{2}\right)\right\rangle\right|^{2} L\left(\mathbf{r}_{2}, \boldsymbol{\rho}_{2}\right) L\left(\mathbf{r}_{1}, \boldsymbol{\rho}_{1}\right) \\
& \quad \times H\left(\boldsymbol{\rho}_{1}, \boldsymbol{\rho}_{2}, \boldsymbol{\rho}_{3}, \boldsymbol{\rho}_{4}\right) L\left(\mathbf{r}_{3}, \boldsymbol{\rho}_{3}\right) L\left(\mathbf{r}_{4}, \boldsymbol{\rho}_{4}\right) \\
& \quad \times\left|\left\langle G\left(\mathbf{r}^{\prime}-\mathbf{r}_{3}\right)\right\rangle\right|^{2}\left|\left\langle G\left(\mathbf{r}-\mathbf{r}_{4}\right)\right\rangle\right|^{2} \mathrm{~d} \mathbf{r}_{1} \ldots \mathrm{d} \mathbf{r}_{4} \mathrm{~d} \boldsymbol{\rho}_{1} \ldots \mathrm{d} \boldsymbol{\rho}_{4},
\end{aligned}
$$

where $\langle G(\mathbf{r})\rangle$ is the mean Green's function of the wave equation; $L\left(\mathbf{r}, \mathbf{r}^{\prime}\right)$ represents a diffusive pair of fields that propagate from $\mathbf{r}$ to $\mathbf{r}^{\prime}$; and the operator $H\left(\boldsymbol{\rho}_{1}, \boldsymbol{\rho}_{2}, \boldsymbol{\rho}_{3}, \boldsymbol{\rho}_{4}\right)$ stands for the diffusion partner exchange. The latter is called a Hikami vertex and reads

$$
\begin{gathered}
H\left(\boldsymbol{\rho}_{1}, \boldsymbol{\rho}_{2}, \boldsymbol{\rho}_{3}, \boldsymbol{\rho}_{4}\right)=\frac{h}{4} \delta\left(\boldsymbol{\rho}_{1}, \boldsymbol{\rho}_{2}, \boldsymbol{\rho}_{3}, \boldsymbol{\rho}_{4}\right) \\
\times(\overbrace{\Delta_{\boldsymbol{\rho}_{1}}+\cdots+\Delta_{\boldsymbol{\rho}_{4}}}^{1}+\overbrace{2 \nabla \boldsymbol{\rho}_{1} \cdot \nabla \boldsymbol{\rho}_{2}}^{2}+\overbrace{2 \nabla \boldsymbol{\rho}_{3} \cdot \nabla \boldsymbol{\rho}_{4}}^{3},,
\end{gathered}
$$

where $\delta\left(\boldsymbol{\rho}_{1}, \boldsymbol{\rho}_{2}, \boldsymbol{\rho}_{3}, \boldsymbol{\rho}_{4}\right)$ means $\int \delta\left(\boldsymbol{\rho}_{1}-\boldsymbol{\rho}\right) \delta\left(\boldsymbol{\rho}_{2}-\boldsymbol{\rho}\right) \delta\left(\boldsymbol{\rho}_{3}-\boldsymbol{\rho}\right)$ $\delta\left(\boldsymbol{\rho}_{4}-\boldsymbol{\rho}\right) \mathrm{d} \boldsymbol{\rho}$ and $h$ is the weight of the vertex defined in Ref. [6], for example. We have labeled three terms in Eq. (E3). In the literature dedicated to mesoscopic physics, it is often argued that the term 1 is negligible (since it forces the crossing to occur at the sample surface; see Ref. [7]), while the two others give rise to equal contributions; see Ref. [8]. This was the approach adopted in Ref. [18], where the Hikami vertex was replaced by twice the term 3 . We call $C_{2}^{\text {out }}$ the analytical form of the correlation calculated in this way. Similarly, we call $C_{2}^{\text {in }}$ the form obtained by keeping twice the term 2. In fact, in the R-T configuration, there is no good reason to neglect the term 1 nor to assume that terms 2 and 3 are of the same amplitude. For this reason, we compute here all contributions explicitly. We write the complete correlator as

$$
C_{2}^{R T}\left(\mathbf{r}, \mathbf{r}^{\prime}\right)=C_{2}^{\Delta}\left(\mathbf{r}, \mathbf{r}^{\prime}\right)+\frac{C_{2}^{\mathrm{in}}\left(\mathbf{r}, \mathbf{r}^{\prime}\right)+C_{2}^{\mathrm{out}}\left(\mathbf{r}, \mathbf{r}^{\prime}\right)}{2},
$$

where the three contributions come from the three terms labeled in the vertex (E3). Following the same approach as in Ref. [18], we find in three-dimensional space

$$
\begin{aligned}
C_{2}^{\Delta}= & \frac{-3}{4 k^{2} \ell^{2}} \int \frac{J_{0}(q \Delta r / L) \operatorname{sh}\left(q z_{0} / L\right)^{2}}{q \operatorname{sh}\left[q\left(1+2 z_{0} / L\right)\right]^{2}} \\
& \times\left(\frac{27 L}{10 \ell} \operatorname{sh}\left[q\left(1+\frac{z_{0}}{L}\right)\right] \operatorname{sh}\left(q \frac{z_{0}}{L}\right)\right. \\
& \left.+\left(\frac{27}{20}+\frac{5 \ell^{2}}{3 L^{2}}\right) q \operatorname{sh}\left[q\left(1+2 \frac{z_{0}}{L}\right)\right]\right) \mathrm{d} q,
\end{aligned}
$$




$$
\begin{aligned}
C_{2}^{\text {in }}= & \frac{-45}{8 k^{2} \ell^{2}} \int \frac{J_{0}(q \Delta r / L) \operatorname{sh}\left(q z_{0} / L\right)^{2}}{q^{2} \operatorname{sh}\left[q\left(1+2 z_{0} / L\right)\right]^{2}} \\
& \times\left(-q \operatorname{ch}\left[q\left(1+\frac{z_{0}}{L}\right)\right]+\operatorname{sh}(q)\right) \mathrm{d} q, \quad \text { (E6) } \\
C_{2}^{\text {out }}= & \frac{-45}{8 k^{2} \ell^{2}} \int \frac{J_{0}(q \Delta r / L) \operatorname{sh}\left(q z_{0} / L\right)^{2}}{q^{2} \operatorname{sh}\left[q\left(1+2 z_{0} / L\right)\right]^{2}} \\
\times & \left(-q \operatorname{ch}(q)+\left[1+q^{2}\left(1+\frac{2 z_{0}}{L}+\frac{2 z_{0}^{2}}{L^{2}}\right)\right] \operatorname{sh}(q)\right) \mathrm{d} q,
\end{aligned}
$$

and in two-dimensional space

$$
\begin{aligned}
C_{2}^{\Delta}= & \frac{-L}{\pi k \ell^{2}} \int \frac{\cos (q \Delta r / L) \operatorname{sh}\left(q z_{0} / L\right)^{2}}{q^{2} \operatorname{sh}\left[q\left(1+2 z_{0} / L\right)\right]^{2}} \\
& \times\left(\frac{16(1+\pi / 2) L}{\pi^{2}(1+\pi / 4) \ell} \operatorname{sh}\left(q \frac{z_{0}}{L}\right) \operatorname{sh}\left[q\left(1+\frac{z_{0}}{L}\right)\right]\right. \\
& \left.+\left(\frac{4(1+\pi / 2)^{2}}{\pi^{2}(1+\pi / 4)}+\frac{(1+\pi / 4) \ell^{2}}{L^{2}}\right) q \operatorname{sh}\left[q\left(1+2 \frac{z_{0}}{L}\right)\right]\right) \mathrm{d} q,
\end{aligned}
$$

$$
\begin{aligned}
C_{2}^{\text {in }}= & \frac{-32 L}{\pi k \ell^{2}} \frac{(1+\pi / 4)}{\pi^{2}} \int \frac{\cos (q \Delta r / L) \operatorname{sh}\left(q z_{0} / L\right)^{2}}{q^{3} \operatorname{sh}\left[q\left(1+2 z_{0} / L\right)\right]^{2}} \\
& \times\left(-q \operatorname{ch}\left[q\left(1+\frac{z_{0}}{L}\right)\right]+\operatorname{sh}(q)\right) \mathrm{d} q, \\
C_{2}^{\text {out }}= & \frac{-32 L}{\pi k \ell^{2}} \frac{(1+\pi / 4)}{\pi^{2}} \int \frac{\cos (q \Delta r / L) \operatorname{sh}\left(q z_{0} / L\right)^{2}}{q^{3} \operatorname{sh}\left[q\left(1+2 z_{0} / L\right)\right]^{2}} \\
& \times\left(-q \operatorname{ch}(q)+\left[1+q^{2}\left(1+\frac{2 z_{0}}{L}+\frac{2 z_{0}^{2}}{L^{2}}\right)\right] \operatorname{sh}(q)\right) \mathrm{d} q,
\end{aligned}
$$

where $z_{0}$ is the extrapolation length (see Ref. [18] for details). We have represented these different contributions in Fig. 12 in the case of wave propagation through a 2D disordered slab. The contributions of $C_{2}^{\text {out }}$ and $C_{2}^{\Delta}$ are negative whereas $C_{2}^{\text {in }}$ is positive. In addition, $C_{2}^{\text {in }}$ and $C_{2}^{\text {out }}$ do not have the same amplitude. However, the sum of all terms, given by Eq. (E4), turns out to be well approximated by $C_{2}^{\text {out }}$, as it was done in Ref. [18]. Both expressions are in good agreement with simulations of microscopic wave propagation. Hence, we conclude that all conclusions of Ref. [18] remain qualitatively valid. In particular, at large optical depth $L \gg \ell$, we find $C_{2}^{R T}(\Delta \mathbf{r})=$ $-f(\Delta r / L) /(k L)^{d-1}$, where $f(x)$ is a positive decaying function of range unity given by

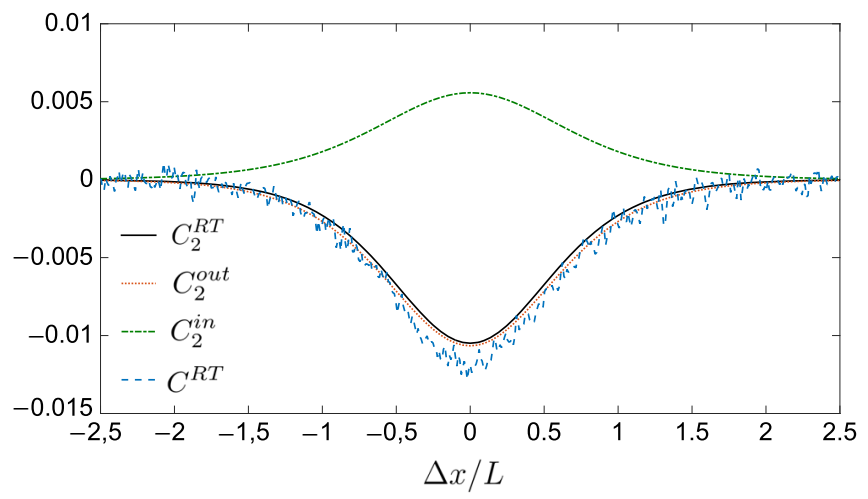

FIG. 12. Analytical predictions for the $C_{2}^{R T}$ correlation (black solid line), $C_{2}^{\text {out }}$ correlation (red dotted line), and $C_{2}^{\text {in }}$ correlation (green dashed-dotted line) compared with the simulation of 2D wave propagation in a disordered medium (blue dashed line). The parameters of the simulations are $k \ell=10, L / \ell=10, \theta_{a}=0$.

$$
\begin{aligned}
f(x)= & \int_{0}^{\infty} \frac{q \cos (q x)}{\operatorname{sh}(q)}\left(\frac{(1+\pi / 2)^{2}+\pi(1+\pi / 2)}{4 \pi(1+\pi / 4)}\right. \\
& \left.+\frac{1+\pi / 4\left[-2 q \operatorname{ch}(q)+\operatorname{sh}(q)\left(2+q^{2}\right)\right]}{\pi}\right) \mathrm{d} q \\
q^{2} \operatorname{sh}(q) &
\end{aligned}
$$

in dimension 2 and by

$$
\begin{aligned}
f(x)= & \int_{0}^{\infty} \frac{q^{2} J_{0}(q x)}{\operatorname{sh}(q)} \\
& \times\left(\frac{21}{20}+\frac{5\left[-2 q \operatorname{ch}(q)+\operatorname{sh}(q)\left(2+q^{2}\right)\right]}{4}\right) \mathrm{d} q
\end{aligned}
$$

in dimension 3. This means, in particular, that the R-T correlation becomes independent of the disorder strength $k \ell$ in the deep diffusive regime.

\section{APPENDIX F: DEPENDENCE ON THE ANGLE OF INCIDENCE IN THE REGIME $L \sim \ell$}

In this appendix, we discuss the angular dependence of the shape of the correlation $C^{R T}(\Delta \mathbf{r})$ in the regime of moderate optical thickness. In Fig. 13, we have represented the result of $3 \mathrm{D}$ numerical simulations of the wave propagation in a disordered slab of optical thickness $L / \ell=$ 1 and disorder strength $k \ell=15$. The horizontal axis is defined as the intersection of the incidence plane with the sample surface (here, $\theta_{a} \simeq 75^{\circ}$ ). As discussed in the main text, the correlation is positive for $\Delta \mathbf{r} \lesssim L$ and presents negative side lobes that are more pronounced along the illumination direction. We have analyzed the angular dependence of this shape along the direction $\Delta y=0$. The results are presented in Fig. 14. For $\theta_{a}=57^{\circ}$, the correlation $C^{R T}(\Delta x)$ is asymmetric. When the angle of incidence $\theta_{a}$ increases, both the positive central peak and the negative side lobes grow. In addition, the correlation becomes more and more symmetric. We interpret the shape 


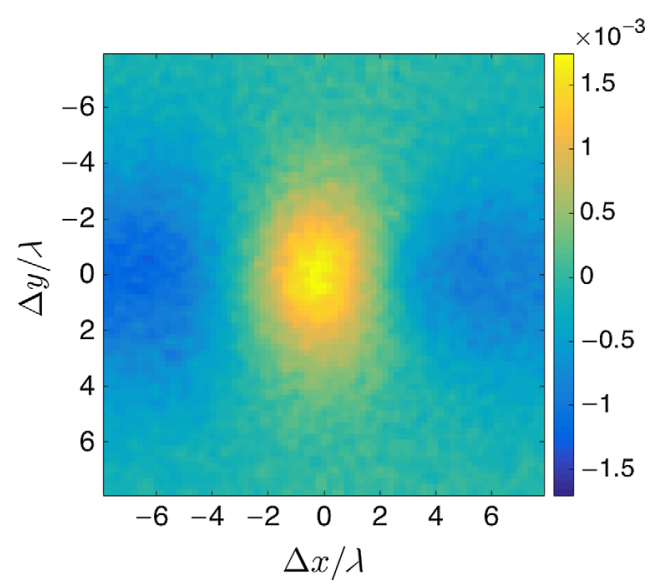

FIG. 13. $\quad C^{R T}(\Delta \mathbf{r})$ calculated from 3D numerical simulations of the wave propagation in a disordered slab of moderate optical depth. The direction $\Delta y=0$ is defined as the intersection of the incidence plane with the sample surface. The parameters are $L / \ell=1, k \ell=15, \theta_{a} \simeq 75^{\circ}$.

of this correlation function as the result of the superposition of two contributions, $C_{2}^{R T}$ and $C_{0}^{R T}$. The contribution $C_{2}^{R T}$ is a negative dip with a minimum located at $\Delta x>0$. As discussed in the main text, this contribution is almost independent of $\theta_{a}$. On the other hand, the contribution $C_{0}^{R T}$ contains both a positive peak located at $\Delta x \simeq 0$ and symmetric negative side lobes. It is also strongly dependent on the illumination angle (see Appendix G for details). Hence, the latter is responsible for the anisotropic shape observed in Fig. 13 and the evolution presented in Fig. 14. In particular, the correlation $C^{R T}(\Delta x)$ shown in Fig. 14 becomes more and more symmetric for increasing $\theta_{a}$, because the amplitude of the negative side lobes of $C_{0}^{R T}$ gets larger than the $C_{2}^{R T}$ dip. A microscopic interpretation of this phenomenon is proposed in the next appendix.

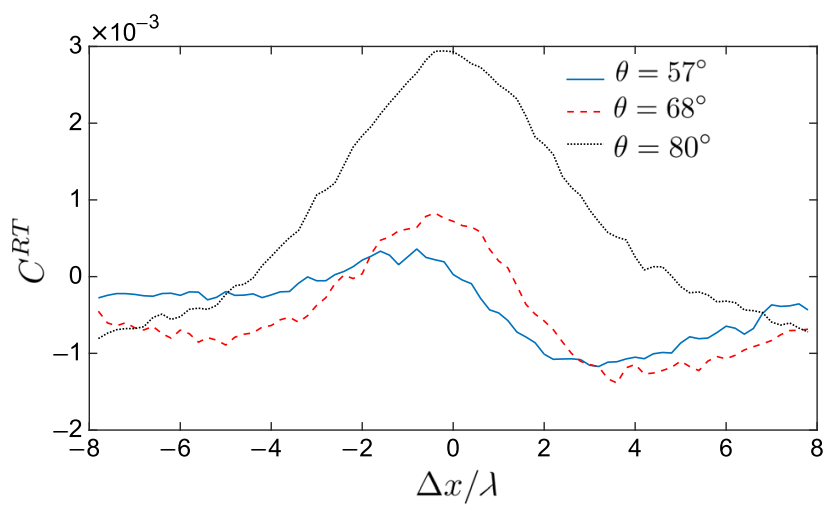

FIG. 14. Dependence of $C^{R T}(\Delta \mathbf{r})$ on the illumination angle $\theta_{a}$, along the illumination direction $\Delta y=0$ (horizontal axis of Fig. 13). The parameters of the $3 \mathrm{D}$ simulation are $L / \ell=1, k \ell=15$.

\section{APPENDIX G: $C_{0}^{R T}$ CORRELATION WITH PLANE WAVE ILLUMINATION}

The $C_{0}$ correlation has first been introduced in Refs. [23,34] in the case of a point source excitation. Here, we consider the same class of scattering processes, but generated by a plane wave excitation. As we will see, both the formal calculation and the qualitative consequences are different from the case of a point source excitation. The microscopic representations of the $C_{0}^{R T}$ diagrams are represented in Fig. 15. Each diagram involves scattering paths that visit a common scatterer located near the front side of the sample. The symmetric diagrams (not shown) that involve a common scatterer at the outputs can be neglected for the same reason as the $C_{1}$ correlation (see Appendix C). Using the same notations as in Appendix E, the four-field correlator $\mathcal{C}_{0}^{R T}(\Delta \mathbf{r})=\left\langle\delta E_{R}(\mathbf{r}) \delta E_{R}(\mathbf{r})^{*} \delta E_{T}\left(\mathbf{r}^{\prime}\right) \delta E_{T}\left(\mathbf{r}^{\prime}\right)^{*}\right\rangle_{C_{0}}$ takes, in $3 \mathrm{D}$, the form

$$
\begin{aligned}
\mathcal{C}_{0}^{R T}(\Delta \mathbf{r})= & \frac{4 \pi}{\ell} \int V\left(\mathbf{r}_{2}, \mathbf{r}_{3}\right) L\left(\mathbf{r}_{2}, \mathbf{r}_{4}\right) L\left(\mathbf{r}_{3}, \mathbf{r}_{5}\right) \\
& \times\left|\left\langle G\left(\mathbf{r}-\mathbf{r}_{4}\right)\right\rangle\right|^{2}\left|\left\langle G\left(\mathbf{r}^{\prime}-\mathbf{r}_{5}\right)\right\rangle\right|^{2} \mathrm{~d} \mathbf{r}_{2} \mathrm{~d} \mathbf{r}_{3} \mathrm{~d} \mathbf{r}_{4} \mathrm{~d} \mathbf{r}_{5},
\end{aligned}
$$

where $V\left(\mathbf{r}_{2}, \mathbf{r}_{3}\right)$ is the sum of the four possibilities for connecting the input plane wave to the ladder diagrams starting in $\mathbf{r}_{2}$ and $\mathbf{r}_{3}$, as represented in Fig. 15. For example, the contribution of Fig. 15(a) to the vertex $V$ is

$$
\begin{aligned}
V^{(a)}\left(\mathbf{r}_{2}, \mathbf{r}_{3}\right)= & \int\left\langle E\left(\mathbf{r}_{2}\right)\right\rangle^{*}\left\langle E\left(\mathbf{r}_{3}\right)\right\rangle^{*}\left|\left\langle E\left(\mathbf{r}_{1}\right)\right\rangle\right|^{2} \\
& \times\left\langle G\left(\mathbf{r}_{2}-\mathbf{r}_{1}\right)\right\rangle\left\langle G\left(\mathbf{r}_{3}-\mathbf{r}_{1}\right)\right\rangle \mathrm{d} \mathbf{r}_{1},
\end{aligned}
$$

(a)

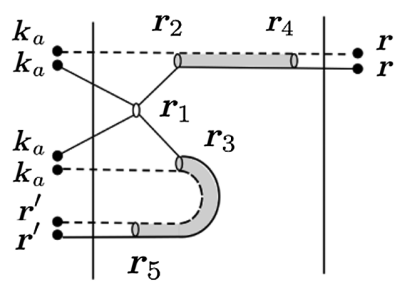

(c)

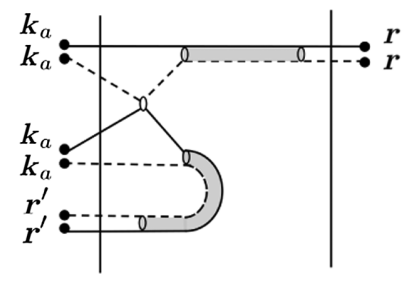

(b)

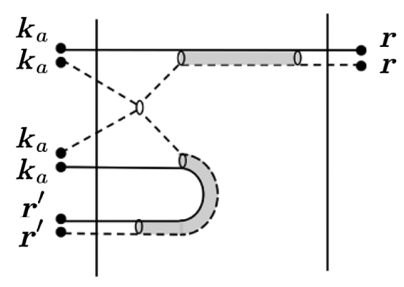

(d)

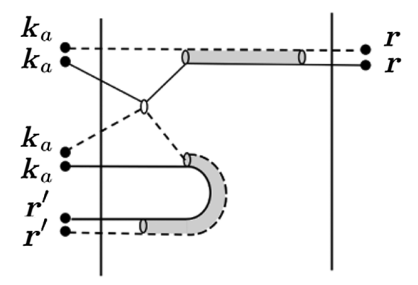

FIG. 15. Leading diagrams contributing to $C_{0}^{R T}$. Shaded tubes represent diffusive paths (ladders); single solid lines stand for averaged fields, and single dashed lines for their complex conjugates. The extra scatterer located near the surface boundary can connect the ladders in four different ways. 
where the mean field $\langle E(\mathbf{r})\rangle$ depends on the incidence angle. By integrating over the transverse coordinates, we get

$$
\begin{aligned}
\mathcal{C}_{0}^{R T}(\Delta \mathbf{r})= & \frac{\ell}{16 \pi^{3}} \int\left(\left[\tilde{V}_{\mathbf{q}_{a}, \mathbf{q}}^{(a)}\left(z_{2}, z_{3}\right)+\tilde{V}_{\mathbf{q}_{a}, \mathbf{q}}^{(c)}\left(z_{2}, z_{3}\right)\right] e^{i\left(\mathbf{q}_{a}-\mathbf{q}\right) \cdot \Delta r}\right. \\
& \left.+\left[\tilde{V}_{\mathbf{q}_{a}, \mathbf{q}}^{(b)}\left(z_{2}, z_{3}\right)+\tilde{V}_{\mathbf{q}_{a}, \mathbf{q}}^{(d)}\left(z_{2}, z_{3}\right)\right] e^{i\left(\mathbf{q}-\mathbf{q}_{a}\right) \cdot \Delta r}\right) \\
& \times L_{\mathbf{q}_{-} \mathbf{q}_{a}}\left(z_{2}, 0\right) L_{\mathbf{q}-\mathbf{q}_{a}}\left(z_{3}, L\right) \mathrm{d} \mathbf{q} \mathrm{d} z_{2} \mathrm{~d} z_{3}, \quad(\mathrm{G} 3)
\end{aligned}
$$

where $z$ labels longitudinal coordinates; $\mathbf{q}_{a}$ is the transverse component of the incident wave vector $\mathbf{k}_{a}$; and $L_{\mathbf{q}}\left(z, z^{\prime}\right)$ is the Fourier transform of $L\left(\mathbf{r}, \mathbf{r}^{\prime}\right)$ with respect to the transverse part of the coordinate $\mathbf{r}-\mathbf{r}^{\prime}$. In addition, the components $\tilde{V}$ are given by

$$
\begin{aligned}
\tilde{V}_{\mathbf{q}_{a}, \mathbf{q}}^{(a)}\left(z_{2}, z_{3}\right)= & \int \bar{G}_{\mathbf{q}_{a}}\left(0, z_{1}\right)^{2} \bar{G}_{2 \mathbf{q}_{a}-\mathbf{q}}\left(z_{1}, z_{3}\right) \\
& \times \bar{G}_{\mathbf{q}}\left(z_{1}, z_{2}\right) \bar{G}_{\mathbf{q}_{a}}\left(0, z_{2}\right)^{*} \bar{G}_{\mathbf{q}_{a}}\left(0, z_{3}\right)^{*} \mathrm{~d} z_{1}, \\
\tilde{V}_{\mathbf{q}_{a}, \mathbf{q}}^{(c)}\left(z_{2}, z_{3}\right)= & \int\left|\bar{G}_{\mathbf{q}_{a}}\left(0, z_{1}\right)\right|^{2} \bar{G}_{\mathbf{q}}\left(z_{1}, z_{3}\right)^{*} \\
& \times \bar{G}_{\mathbf{q}}\left(z_{1}, z_{2}\right) \bar{G}_{\mathbf{q}_{a}}\left(0, z_{2}\right)^{*} \bar{G}_{\mathbf{q}_{a}}\left(0, z_{3}\right), \mathrm{d} z_{1},
\end{aligned}
$$

$\tilde{V}^{(b)}=\tilde{V}^{(a) *}$, and $\tilde{V}^{(c)}=\tilde{V}^{(d) *}$. In these expressions, $\bar{G}_{\mathbf{q}}\left(z, z^{\prime}\right)=i e^{i k_{z}\left(z^{\prime}-z\right)} e^{-\left|z^{\prime}-z\right| / 2 \mu \ell} /\left(2 k_{z}\right)$, with $k_{z}=\sqrt{k^{2}-q^{2}} \equiv$ $k \mu$, is the transverse Fourier transform of the mean Green's function of the Helmholtz equation. We now make the approximations $L\left(z_{2}, 0, q-q_{a}\right) \simeq L\left(0,0, q-q_{a}\right)$ and $L\left(z_{3}, L, q-q_{a}\right) \simeq L\left(0, L, q-q_{a}\right), \quad$ and we integrate Eq. (G3) over the longitudinal coordinates $z_{1}, z_{2}$, and $z_{3}$. The correlator becomes

$$
\begin{aligned}
\mathcal{C}_{0}^{R T}(\Delta \mathbf{r})= & \frac{\ell^{4}}{128 \pi^{3} k^{6}} \int \cos \left[\left(\mathbf{q}_{a}-\mathbf{q}\right) \cdot \Delta \mathbf{r}\right] \\
& \times L_{\mathbf{q}_{-} \mathbf{q}_{a}}(0,0) L_{\mathbf{q}-\mathbf{q}_{a}}(0, L) F\left(\mu, \mu_{a}, k \ell\right) \mathrm{d} \mathbf{q},
\end{aligned}
$$

with

$$
\begin{aligned}
F\left(\mu, \mu_{a}, k \ell\right)= & \frac{2\left(\mu_{a}-\mu\right)}{\mu_{a}\left(2 \mu_{a}-\mu\right)\left(\mu+\mu_{a}\right)}\left[9 \mu^{3}+18 \mu^{2} \mu_{a}\right. \\
& \left.+11 \mu \mu_{a}^{2}+2 \mu_{a}^{3}+4 \mu^{3} \mu_{a}^{2}\left(\mu-\mu_{a}\right)^{2} k^{2} \ell^{2}\right] \\
& /\left(\left[9 \mu^{2}+\mu_{a}^{2}+6 \mu \mu_{a}+4 \mu^{2} \mu_{a}^{2}\left(\mu-\mu_{a}\right)^{2} k^{2} \ell^{2}\right]\right. \\
& \left.\times\left[\left(\mu+\mu_{a}\right)^{2}+4 \mu^{2} \mu_{a}^{2}\left(\mu-\mu_{a}\right)^{2} k^{2} \ell^{2}\right]\right),
\end{aligned}
$$

where $\mu_{a}=\sqrt{1-q_{a}^{2} / k^{2}}$ and $\mu=\sqrt{1-q^{2} / k^{2}}$.

Normalizing the correlator by the intensity product $\left\langle\left|\delta E_{R}(\mathbf{r})\right|^{2}\right\rangle\left\langle\left|\delta E_{T}\left(\boldsymbol{r}^{\prime}\right)\right|^{2}\right\rangle=\ell / 6 k^{4} \mu_{a}^{2} L$ and the integration momentum variable by the sample thickness $L$, we finally obtain

$$
\begin{aligned}
C_{0}^{R T}(\Delta \mathbf{r})= & \frac{27 \mu_{a}^{2} k L}{4 \pi(k \ell)^{3}} \int \cos \left[\frac{\left(\mathbf{q}_{a}^{\prime}-\mathbf{q}^{\prime}\right) \cdot \Delta \boldsymbol{r}}{L}\right] \\
& \times P\left(\mathbf{q}_{a}^{\prime}, \mathbf{q}^{\prime}\right) F\left(\mu, \mu_{a}, k \ell\right) \mathrm{d} \mathbf{q}^{\prime},
\end{aligned}
$$

where $q_{a}^{\prime}=q_{a} L=k L \sin \theta_{a}$, and the ladder contribution $P\left(\mathbf{q}_{a}^{\prime}, \mathbf{q}^{\prime}\right)$ is defined as

$$
P\left(\mathbf{q}_{a}^{\prime}, \mathbf{q}^{\prime}\right)=\frac{\operatorname{sh}\left[\left|\mathbf{q}_{a}^{\prime}-\mathbf{q}^{\prime}\right| z_{0} / L\right]^{3} \operatorname{sh}\left[\left|\mathbf{q}_{a}^{\prime}-\mathbf{q}^{\prime}\right|\left(1+z_{0} / L\right)\right]}{\left|\mathbf{q}_{a}^{\prime}-\mathbf{q}^{\prime}\right|^{2} \operatorname{sh}\left[\left|\mathbf{q}_{a}^{\prime}-\mathbf{q}^{\prime}\right|\left(1+2 z_{0} / L\right)\right]^{2}} .
$$

In order to obtain the result (G8) we used various approximations that are justified in the diffusive regime $L>\ell$ only. Therefore, we must be cautious not to use this result in the quasiballistic regime $L \lesssim \ell$. We note also that in the deep diffusive regime $L \gg \ell, z_{0}$ and for small angle of incidence $\left(\mu_{a} \simeq 1\right)$, the $C_{0}^{R T}$ correlation with plane wave illumination takes the compact form

$C_{0}^{R T}(\Delta \mathbf{r}) \simeq \frac{5}{16 \pi k^{4} L^{4}} \int \cos \left[\frac{\left(\mathbf{q}_{a}^{\prime}-\mathbf{q}^{\prime}\right) \cdot \Delta \mathbf{r}}{L}\right] \frac{q^{\prime 2}\left|\mathbf{q}_{a}^{\prime}-\mathbf{q}^{\prime}\right|}{\operatorname{sh}\left[\left|\mathbf{q}_{a}^{\prime}-\mathbf{q}^{\prime}\right|\right]} \mathrm{d} \mathbf{q}^{\prime}$,

which scales as $C_{0}^{R T} \propto 1 /(k L)^{4}$. In this regime, it is, therefore, much smaller than $C_{2}^{R T} \propto 1 /(k L)^{2}$ (see Appendix E). This explains why it is not observed experimentally in the diffusive regime.

Before analyzing the result (G8) in more detail, let us comment on the differences with the correlation calculated with plane wave outputs or point-source inputs. For planewave outputs, we find

$$
C_{0}^{R T}\left(\mathbf{k}_{b}, \mathbf{k}_{b^{\prime}}\right)=\int \frac{\mathrm{d} \Delta \mathbf{r}}{\mathcal{A}} C_{0}^{R T}(\Delta \mathbf{r})=0,
$$

for all observation directions $\mathbf{k}_{b}, \mathbf{k}_{b^{\prime}}$. This striking result comes from the fact that $F\left(\mu_{a}, \mu_{a}, k \ell\right)=0$. This means that, for plane wave outputs, the diagram of Fig. 15(a) [Fig. 15(b)] is compensated by the one in Fig. 15(c) [Fig. 15(d)]. This result turns out to be completely different from the one obtained in the configuration involving pointlike sources and detectors, where the diagrams of Figs. 15(a) and 15(b) do not contribute to the correlation.

Let us now discuss the strong dependence of the result (G8) on the illumination angle $\theta_{a}$. At the origin of this dependence is the total momentum conservation during the interaction with the common scatterer of Fig. 15. The information carried by the illumination plane wave is transmitted to the ladder diagrams that conserve momentum over long distances, so that the input information finally reaches the sample boundaries. The same property occurs in the well-known memory effect introduced in 

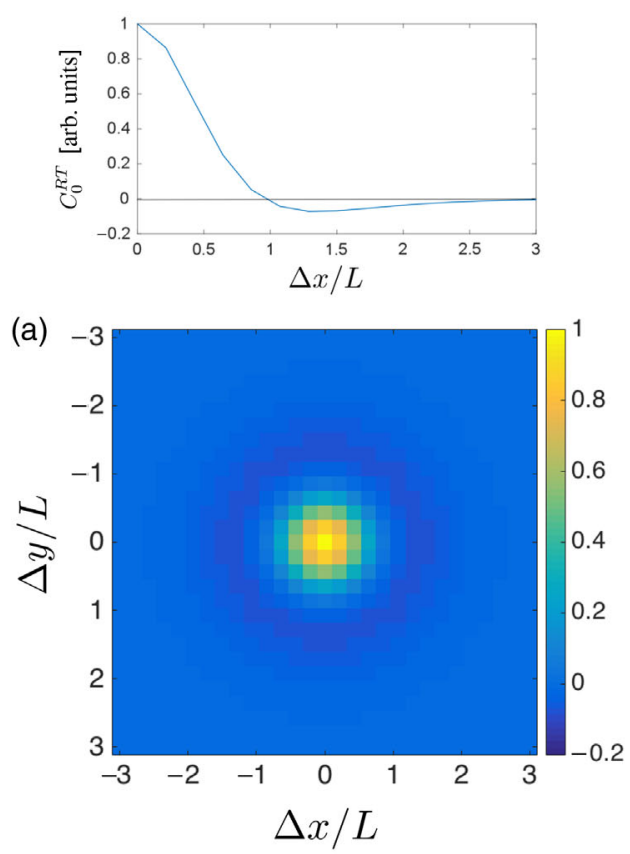
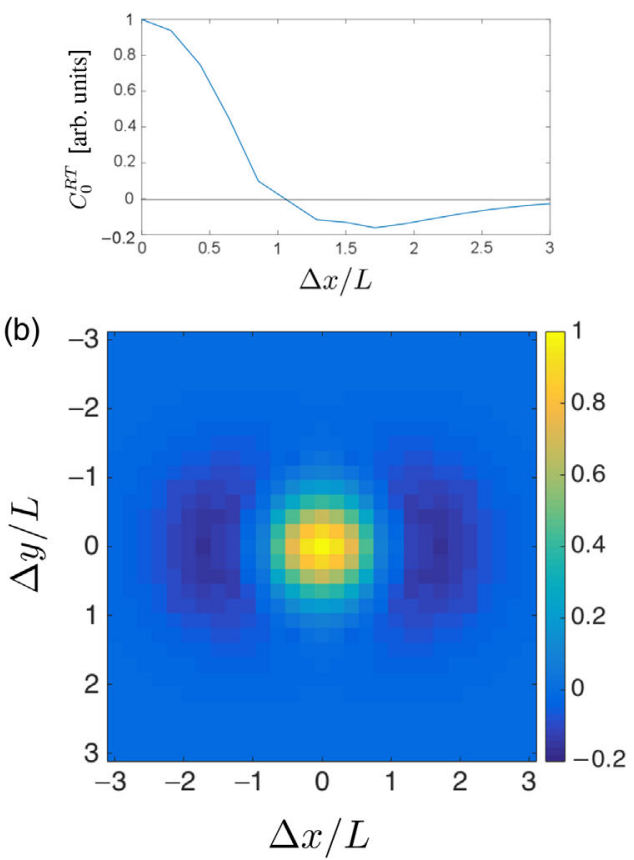

FIG. 16. Analytical prediction for the $C_{0}^{R T}$ correlation (normalized to unity) represented in Fig. 15 for two different illumination angles, (a) $\theta_{a}=0$ and (b) $\theta_{a}=45^{\circ}$, and their cut (top figure) along the direction $\Delta y=0$, which is defined as the intersection of the incidence plane with the sample surface. The parameters are $\lambda=632 \mathrm{~nm}, L=50 \mu \mathrm{m}, \ell=15 \mu \mathrm{m}$. The diffusive approximation was used for the ladder diagrams.

Ref. [15]. We illustrate the dependence of the correlation (G8) on $\theta_{a}$ in Fig. 16. For $\theta_{a}=0$, the rotational symmetry is preserved so that $C_{0}^{R T}(\Delta \mathbf{r})$ depends on $\Delta r$ only. It presents a positive peak centered in $\Delta r=0$ that extends over a distance $\Delta r \sim L$. Beyond this distance, the correlation presents small negative side lobes, which are such that the sum rule (G11) is satisfied. When the rotational symmetry is broken $\left(\theta_{a} \neq 0\right)$, the correlation becomes anisotropic. It now presents two mirror symmetries with respect to the intersection of the incidence plane with the sample surface. The direction of this intersection defines the horizontal axis in Fig. 16. We observe that the negative side lobes become more pronounced along this direction. In addition, the amplitude of both the central peak and the side lobes gets larger for increasing $\theta_{a}$, in agreement with the sum rule (G11), as well as the numerical observations discussed in the previous appendix.

The previous analysis shows that $C_{0}^{R T}$ reproduces the features observed experimentally in the R-T correlation in the regime $L \sim \ell$. As was observed in the experiment, $C_{0}^{R T}$ is long range, keeps a memory on the incidence angle, becomes anisotropic for $\theta_{a} \neq 0$, and presents a central peak and negative side lobes. Both the peak and the side lobes become more pronounced when $\theta_{a}$ is increased. That said, it should be stressed that the formula (G8) does not reproduce quantitatively the amplitude of the positive correlation observed in the regime $L \sim \ell$. This is not much of a surprise since, as we explained above, the result (G8) was obtained in the diffusive regime $L>\ell$. In addition, it is worth mentioning that the scattering processes described by $C_{0}^{R T}$ and $C_{2}^{R T}$ are not the only ones that contribute in the quasiballistic regime $L \lesssim \ell$. For example, it is clear that, in the regime $L \ll \ell$, the scattering sequences where the four fields interact with a common scatterer play an important role as well. We did not discuss such contributions to the correlation in the main text because the regime $L \ll \ell$ is not probed experimentally and such scattering sequences do not possess the dependence on the illumination angle mentioned above.

[1] P. Sebbah, Waves and Imaging through Complex Media (Springer, Netherlands, 1999).

[2] B. Shapiro, Large Intensity Fluctuations for Wave Propagation in Random Media, Phys. Rev. Lett. 57, 2168 (1986).

[3] I. Freund, Looking through Walls and around Corners, Physica A 168, 49 (1990).

[4] S. M. Popoff, G. Lerosey, R. Carminati, M. Fink, A. C. Boccara, and S. Gigan, Measuring the Transmission Matrix in Optics: An Approach to the Study and Control of Light Propagation in Disordered Media, Phys. Rev. Lett. 104, 100601 (2010).

[5] A. P. Mosk, A. Lagendijk, G. Lerosey, and M. Fink, Controlling Waves in Space and Time for Imaging and Focusing in Complex Media, Nat. Photonics 6, 283 (2012).

[6] R. Berkovits and S. Feng, Correlations in Coherent Multiple Scattering, Phys. Rep. 238, 135 (1994). 
[7] M. C. W. van Rossum and T. M. Nieuwenhuizen, Multiple Scattering of Classical Waves: Microscopy, Mesoscopy, and Diffusion, Rev. Mod. Phys. 71, 313 (1999).

[8] E. Akkermans and G. Montambeaux, Mesoscopic Physics of Electrons and Photons (Cambridge University Press, Cambridge, England, 2007).

[9] S. Feng, C. Kane, P. A. Lee, and A. D. Stone, Correlations and Fluctuations of Coherent Wave Transmission through Disordered Media, Phys. Rev. Lett. 61, 834 (1988).

[10] A. Z. Genack, N. Garcia, and W. Polkosnik, Long-Range Intensity Correlation in Random Media, Phys. Rev. Lett. 65, 2129 (1990).

[11] J. F. de Boer, M. P. van Albada, and A. Lagendijk, Transmission and Intensity Correlations in Wave Propagation through Random Media, Phys. Rev. B 45, 658 (1992).

[12] F. Scheffold and G. Maret, Universal Conductance Fluctuations of Light, Phys. Rev. Lett. 81, 5800 (1998).

[13] J. Bertolotti, E. G. van Putten, C. Blum, A. Lagendijk, W. L. Vos, and A.P. Mosk, Non-invasive Imaging through Opaque Scattering Layers, Nature (London) 491, 232 (2012).

[14] O. Katz, P. Heidmann, M. Fink, and S. Gigan, Non-invasive Single-Shot Imaging through Scattering Layers and around Corners via Speckle Correlations, Nat. Photonics 8, 784 (2014).

[15] I. Freund, M. Rosenbluh, and S. Feng, Memory Effects in Propagation of Optical Waves through Disordered Media, Phys. Rev. Lett. 61, 2328 (1988).

[16] D. B. Rogozkin and M. Y. Cherkasov, Long-range Intensity Correlations in Wave Reflection from a Disordered Medium, Phys. Rev. B 51, 12256 (1995).

[17] L. S. Froufe-Pérez, A. Garcia-Martin, G. Cwilich, and J. J. Saenz, Fluctuations and Correlations in Wave Transport through Complex Media, Physica A 386, 625 (2007).

[18] N. Fayard, A. Cazé, R. Pierrat, and R. Carminati, Intensity Correlations between Reflected and Transmitted Speckle Patterns, Phys. Rev. A 92, 033827 (2015).

[19] B. Judkewitz, R. Horstmeyer, I. M. Vellekoop, I. N. Papadopoulos, and C. Yang, Translation Correlations in Anisotropically Scattering Media, Nat. Phys. 11, 684 (2015).

[20] S. Rotter and S. Gigan, Light Fields in Complex Media: Mesoscopic Scattering Meets Wave Control, Rev. Mod. Phys. 89, 015005 (2017).

[21] H. Yu, J. Park, K. Lee, J. Yoon, K. Kim, S. Lee, and Y. Park, Recent Advances in Wavefront Shaping Techniques for Biomedical Applications, Curr. Appl. Phys. 15, 632 (2015).

[22] J. W. Goodman, Speckle Phenomena in Optics (Roberts and Company, Colorado, 2007).

[23] B. Shapiro, New Type of Intensity Correlation in Random Media, Phys. Rev. Lett. 83, 4733 (1999).
[24] P. Sebbah, R. Pnini, and A. Z. Genack, Field and Intensity Correlation in Random Media, Phys. Rev. E 62, 7348 (2000).

[25] T. Strudley, T. Zehender, C. Blejean, E. P. A. M. Bakkers, and O. L. Muskens, Mesoscopic Light Transport by Very Strong Collective Multiple Scattering in Nanowire Mats, Nat. Photonics 7, 413 (2013).

[26] M. Lax, Multiple Scattering of Waves. II. The Effective Field in Dense Systems, Phys. Rev. 85, 621 (1952).

[27] K. Vynck, R. Pierrat, and R. Carminati, Polarization and Spatial Coherence of Electromagnetic Waves in Uncorrelated Disordered Media, Phys. Rev. A 89, 013842 (2014).

[28] D. B. Rogozkin and M. Y. Cherkasov, Long-range Intensity Correlations in a Disordered Medium, Phys. Lett. A 214, 292 (1996).

[29] J. J. Sáenz, L. S. Froufe-Pérez, and A. García-Martín, Intensity Correlations and Fluctuations of Waves Transmitted through Random Media, in Wave Scattering in Complex Media: From Theory to Applications, edited by B. van Tiggelen and S. Skipetrov, NATO Science Series II Vol. 107 (Kluwer, Dordrecht, 2003), p. 175.

[30] A. Zyuzin and B. Spivak, Zh. Eksp. Teor. Fiz. 93, 994 (1987) [Langevin Description of Mesoscopic Fluctuations in Disordered Media, JETP Lett. 66, 560 (1987)].

[31] R. Pnini and B. Shapiro, Fluctuations in Transmission of Waves through Disordered Slabs, Phys. Rev. B 39, 6986 (1989).

[32] N. Fayard, A. Goetschy, R. Pierrat, and R. Carminati, Mutual Information between Reflected and Transmitted Speckle Images, Phys. Rev. Lett. 120, 073901 (2018).

[33] W. K. Hildebrand, A. Strybulevych, S. E. Skipetrov, B. A. van Tiggelen, and J. H. Page, Observation of Infinite-Range Intensity Correlations above, at, and below the Mobility Edges of the 3D Anderson Localization Transition, Phys. Rev. Lett. 112, 073902 (2014).

[34] S. E. Skipetrov and R. Maynard, Nonuniversal Correlations in Multiple Scattering, Phys. Rev. B 62, 886 (2000).

[35] A. Cazé, R. Pierrat, and R. Carminati, Near-Field Interactions and Nonuniversality in Speckle Patterns Produced by a Point Source in a Disordered Medium, Phys. Rev. A 82, 043823 (2010).

[36] O. S. Ojambati, J. T. Hosmer-Quint, K.-J. Gorter, A. P. Mosk, and W. L. Vos, Controlling the intensity of light in large areas at the interfaces of a scattering medium, Phys. Rev. A 94, 043834 (2016).

[37] C. W. Hsu, S. F. Liew, A. Goetschy, H. Cao, and A. D. Stone, Correlation-enhanced control of wave focusing in disordered media, Nat. Phys. 13, 497 (2017).

[38] https://doi.org/10.24378/exe.243.

[39] P. Sebbah, B. Hu, A. Z. Genack, R. Pnini, and B. Shapiro, Spatial-field correlation: The building block of mesoscopic fluctuations, Phys. Rev. Lett. 88, 123901 (2002). 